Y. Okabe

Japanese J. of Math., Vol. 41

1973 p. $69 \sim$ p. 122

\title{
Stationary Gaussian processes with Markovian property and M. Sato's hyperfunctions ${ }^{1)}$
}

\author{
By Yasunori OKABE
}

(Received September 4, 1972)

\section{§1. Introduction}

Let $\boldsymbol{X}=(X(t) ; t \in \boldsymbol{R})$ be a real stationary Gaussian process on a probability space $(\Omega, F, P)$ such that $X(t)$ is continuous in the mean and its expectation is zero. ${ }^{2)}$ For each open set $D$ in $\boldsymbol{R}$, let $\boldsymbol{F}(D)$ denote the smallest sub- $\sigma$-field of $\boldsymbol{F}$ for with all $X(t)(t \in D)$ are measurable. We then define three sub- $\sigma$-fields of $\boldsymbol{F}$ by $\partial \boldsymbol{F}(0)=\bigcap_{\varepsilon>0} \boldsymbol{F}((-\varepsilon, \varepsilon)), \boldsymbol{F}^{+}(0)=\boldsymbol{F}((0, \infty))$ and $\boldsymbol{F}^{-}(0)=$ $\boldsymbol{F}((-\infty, 0))$ which are said to be the germ field, the future field and the past field, respectively. Following K. Urbanik [26] and N. Levinson-H. P. McKean, Jr. [14], we say that the process $\boldsymbol{X}$ has the Markovian property if the future is independent of the past under the condition that the germ is known : in notation,

$$
\boldsymbol{F}^{-}(0) \Perp \boldsymbol{F}^{+}(0) \quad \text { under } \quad \partial \boldsymbol{F}(0) .
$$

In this paper we shall characterize the Markovian property from an operator-theoretical point of view and then investigate the structure of the process $\boldsymbol{X}$ having such Markovian property.

Throughout this section we assume that $\boldsymbol{X}$ is purely non-deterministic. Then it follows from K. Karhunen's representation theorem ([11]) that there exist a real $L^{2}$-function $E$ vanishing on the negative axis and a Brownian motion $(B(t) ; t \in \boldsymbol{R})$ such that for any $t \in \boldsymbol{R}$

$$
X(t)=\int_{R} \frac{1}{\sqrt{2 \pi}} E(t-s) d B(s) \text { and } F((-\infty, t))=\sigma\left(B\left(s_{1}\right)-B\left(s_{2}\right) ; s_{1}, s_{2}<t\right){ }^{3)}
$$

Such a representation kernel $\sqrt{2 \pi}^{-1} E$ is said to be canonical. We denote by $h$ the inverse Fourier transform of $E$ :

1) This study is a continuation of [16] and supported in part by Sakkôkai Foundation.

2) The process $\boldsymbol{X}$ has a separable and measurable version.

3) For any set $\Lambda$ of random variables on $\Omega$, we denote by $\sigma(\Lambda)$ the smallest sub- $\sigma$ field of $\boldsymbol{F}$ for which all elements of $\Lambda$ are measurable. 


$$
h(\lambda)=\tilde{E}(\lambda) \equiv \frac{1}{2 \pi} \int_{R} e^{i \lambda t} E(t) d t, \quad \lambda \in \boldsymbol{R}^{4}{ }^{4)}
$$

It is easy to see that $\boldsymbol{X}$ has a symmetric spectral density $\Delta(\lambda)$ given by $\Delta(\lambda)=|h(\lambda)|^{2}$.

The simplest example of those processes having the Markovian property is the so-called Ornstein-Uhlenbeck Brownian motion with the spectral density $\Delta(\lambda)=c\left(1+\lambda^{2}\right)^{-1}$, where $c$ is a positive constant. In fact, it is easy to verify that

$$
\begin{aligned}
& h(-\lambda)=\sqrt{c}(1+i \lambda)^{-1}, \quad \lambda \in \boldsymbol{R}, \\
& \boldsymbol{F}^{-}(0) \Perp \boldsymbol{F}^{+}(0) \quad \text { under } \quad \sigma(X(0)) .
\end{aligned}
$$

Since $X(0)$ is $\partial F(0)$-measurable, it can be shown that (1.5) implies (1.1). More generally, J. L. Doob [5] has treated those processes having the "finitely multiple" Markovian property and proved that the following two conditions ((1.6) and (1.7)) are equivalent: Given an integer $N \in N$,

(1.6) $\Delta(\lambda)$ is an inverse of a polynomial of degree $2 N$;

(1.7) (i ) $X(t)$ is differentiable up to $N-1$ times in the mean but not differentiable $N$ times in the mean,

(ii) $\boldsymbol{F}^{-}(0) \Perp \boldsymbol{F}^{+}(0)$ under $\sigma\left(X^{(n)}(0) ; n=0,1, \cdots, N-1\right)$.

Also in this case, (1.1) follows from (1.7) (ii) since $\sigma\left(X^{(n)}(0) ; n=0,1, \ldots\right.$, $N-1)$ is contained in $\partial F(0)$. For later use, we note that (1.6) is valid if and only if

(1.8) $h(-\lambda)$ is an inverse of a polynomial $P(\lambda)$ of $\operatorname{order} N, \quad \lambda \in \boldsymbol{R}$.

J. L. Doob has obtained his results showing that (1.6) is equivalent to the fact that $X(t)$ satisfies a stochastic differential equation

$$
P\left(\frac{1}{i} \frac{d}{d t}\right) X(t)=\sqrt{2 \pi} B^{\prime}(t),
$$

where $\left(B^{\prime}(t) ; t \in \boldsymbol{R}\right)$ means a white noise ([10]). In fact, it follows from (1.3) and (1.8) that

$$
P\left(\frac{1}{i} \frac{d}{d t}\right) E=2 \pi \delta \text { in the sense of L. Schwartz's distributions. }
$$

Therefore, combining (1.2) and (1.10), we get (1.9).

4) $\hat{f}$ and $\tilde{f}$ mean the Fourier transform and inverse Fourier transform of $f$, respectively; $\hat{f}(\lambda)=\int_{R} e^{-i \lambda t} f(t) d t$. 
After J. L. Doob, K. Urbanik [26] has given an interesting example $\boldsymbol{X}$ having the "infinitely multiple" Markovian property as follows :

$$
\Delta(\lambda)=c\left(\prod_{n=1}^{\infty}\left(1+\frac{\lambda^{2}}{c_{n}^{2}}\right)\right)^{-1}, \quad \lambda \in \boldsymbol{R}
$$

where $c$ is a positive constant and $\left(\mathrm{c}_{n}\right)_{n=1}^{\infty}$ is a positive increasing sequence such that $\sum_{n=1}^{\infty} \frac{1}{c_{n}}<\infty$. For such a process $X$, he has proved that $X(t)$ is infinitely differentiable in the mean and

$$
\boldsymbol{F}^{-}(0) \Perp \boldsymbol{F}^{+}(0) \quad \text { under } \quad \sigma\left(X^{(n)}(0) ; n \in \boldsymbol{N}_{*}\right),
$$

where $N_{*}=N \cup\{0\} . \quad$ Again, (1.12) yields (1.1) since $\sigma\left(X^{(n)}(0) ; n \in N_{*}\right)$ is contained in $\partial \boldsymbol{F}(0)$. Furthermore we know that $h$ in (1.3) satisfies

$$
h(-\lambda)=\sqrt{c}\left(\prod_{n=1}^{\infty}\left(1+\frac{i \lambda}{c_{n}}\right)\right)^{-1}, \quad \lambda \in \boldsymbol{R} .
$$

Finally, N. Levinson and H. P. McKean, Jr. [14] have given a complete characterization of the Markovian property: the process $\boldsymbol{X}$ has the Markovian property if and only if

(1.14) $h(-\lambda)$ is an inverse of an entire function $P(\lambda)$ of infra-exponential type,,$^{5)} \lambda \in \boldsymbol{R}$.

It is shown from (1.4), (1.8) and (1.13) that the preceding examples satisfy condition (1.14).

We shall explain the method of $K$. Urbanik and N. Levinson-H. P. McKean, Jr. For each open set $D$ in $\boldsymbol{R}$, let $\boldsymbol{M}(D)$ denote the closed subspace of $L^{2}(\Omega, F, P)$ generated by the linear hull of $\{X(t) ; t \in D\}$. In particular, we set $\boldsymbol{M}=\boldsymbol{M}(\boldsymbol{R})$. Furthermore, let $\partial \boldsymbol{M}(0)$ and $\boldsymbol{M}^{-}(0)$ be the closed subspaces of $\boldsymbol{M}$ defined by $\partial \boldsymbol{M}(0)=\bigcap_{\varepsilon>0} \boldsymbol{M}((-\varepsilon, \varepsilon))$ and $\boldsymbol{M}^{-}(0)=\boldsymbol{M}((-\infty, 0))$. Then, since $\boldsymbol{X}$ is a Gaussian process, it is shown that (1.1) is valid if and only if (1.15) the projection of $X(t)$ upon $\boldsymbol{M}^{-}(0)$ belongs to $\partial \boldsymbol{M}(0)$ for any $t>0$.

In order to treat the case of the infinitely multiple Markovian property, K. Urbanik and N. Levinson-H. P. McKean, Jr. have reformulated (1.1) in terms of a real Hilbert space $Z_{\Delta}$ of $L^{2}(\boldsymbol{R}, \Delta(\lambda) d \lambda)$ obtained by closing the linear hull of $\left\{e^{i t \cdot} ; t \in \boldsymbol{R}\right\}$. It has been known that there exists a unitary operator from $\boldsymbol{M}$ onto $Z_{\Delta}$ under which $e^{i t}$ corresponds to $X(t)(t \in \boldsymbol{R})$. Therefore, denoting by $\partial Z_{\Delta}(0)$ (resp. $Z_{\Delta}^{-}(0)$ ) the closed subspace of $Z_{\Delta}$ correspond-

5) See $\S 3$ for the definition of entire function of infra-exponential type. 
ing to $\partial M(0)$ (resp. $M^{-}(0)$ ), we see from (1.15) that (1.1) is valid if and only if (1.61) the projection of $e^{i t \cdot}$ upon $Z_{\Delta}^{-}(0)$ belongs to $\partial Z_{\Delta}(0)$ for any $t>0$.

K. Urbanik and N. Levinson-H. P. McKean Jr. have obtained their results observing that condition (1.16) has an intimate relation to a special Bernstein's problem about weighted polynomial or trigonometrical approximations.

The spirit in this paper is to analyse the process $\boldsymbol{X}$ directly from the standpoint of operator theory as J. L. Doob has done in [5]. Given any polynomial $P(\cdot)$ we have a differential operator $P\left(\frac{1}{i} \frac{d}{d t}\right)$ of finite order with constant coefficients which acts as a local operator in the space $\mathscr{D}^{\prime}$ of L. Schwartz's distributions ([23]). Given any entire function $P(\cdot)$ we might consider a formal differential operator $P\left(\frac{1}{i} \frac{d}{d t}\right)$ of infinite order with constant coefficients. However, it does not always act in $\mathscr{D}^{\prime}$ even if $P(\cdot)$ is of infra-exponential type. Therefore, in order to dispose of the most general case, we need a concept of more general functions than L. Schwartz's distributions. Fortunately, in the space $\mathscr{B}$ of hyperfunctions created by M. Sato ([18], [19], [20]), we can regard $P\left(\frac{1}{i} \frac{d}{d t}\right)$ as a local operator for any entire function $P(\cdot)$ of infra-exponential type. This is the reason why we will use M. Sato's hyperfunctions instead of L. Schwartz's distributions.

Now, we state a detailed content of this paper. From $\S 2$ to $\S 6$, we are concerned with the problem of characterizing the Markovian property; we realize the above stated ideas, reveal the mechanism of the results in [14] and discuss an open problem in H. Dym and H. P. McKean, Jr. [6].

In $\S 2$ we shall define a real Hilbert space $K$ having the correlation function as its reproducing kernel, which is a realization of the space $\boldsymbol{M}$. Furthermore we shall introduce a unitary operator $K$ from $K$ on $L^{2}(R)^{6)}$ having $\check{h}^{-1}{ }^{7)}$ in (1.3) as its symbol. Then, we shall show that the Markovian property of the process $\boldsymbol{X}$ implies the local property of the operator $K$ (Theorem 2.1).

In $\S 3$ we shall review the theory of hyperfunctions and Fourier hyperfunctions due to M. Sato ([18], [19], [20]) and T. Kawai ([12]). As an application, we shall give a remark as to the relationship between the $L^{2}$ functions $E$ and $h$ in (1.3) from the standpoint of Fourier transformations in the space of Fourier hyperfunctions.

6) We denote by $L^{2}(R)$ (resp. $L^{2}$ ) the space of real (resp. complex) valued square integrable functions on $R$.

7) $\breve{f}$ stands for the reflection of $f$ at the origin. 
In $\S 4$ we shall prove, by using the idea of L. de Branges [3] and the theory of hyperfunctions, that the operator $K$ has the local property if and only if its symbol $\check{h}^{-1}$ is an entire function of infra-exponential type (Theorems 4.2 and 4.3). As an application of Theorem 4.3, we shall give a simple proof of the fact ([6], [14]) that if a function $\varphi \in Z_{\Delta}$ can be extended to an entire function of infra-exponential type, then $\varphi \in \partial Z_{\Delta}(0)$, that is, it can be approximated in $Z_{\Delta}$ by trigonometrical functions $\varphi_{n} \in Z_{\Delta}$ such that each $\varphi_{n}$ has the form $\sum_{k=0}^{l_{n}} c_{k}^{n} e^{i t_{k}^{n}}, c_{k}^{n} \in \boldsymbol{R}$ and $\left|t_{k}^{n}\right|<\frac{1}{n}$.

In $\S 5$ we shall show that if the symbol $\check{h}^{-1}$ of $K$ is an entire function of infra-exponential type, then $\boldsymbol{X}$ has the Markovian property with the aid of Fourier transformations in the space of Fourier hyperfunctions. Combining this with Theorems 2.1 and 4.2, it will be found that the Markovian property of $\boldsymbol{X}$, the local property of $K$ and the fact the symbol $\check{h}^{-1}$ of $K$ is an entire function of infra-exponential type are equivalent each other (Theorem 5.2). As an application of our proof in Theorem 5.2, we shall get a concrete representation of the space $\partial Z_{\Delta}(0)$ in case $\boldsymbol{X}$ has the Markovian property. Finally, in the same case, we shall obtain a diagram involving the space $K$ and the space of Fourier hyperfunctions.

In $\S 6$, by introducing the hyperprocess $\mu_{\boldsymbol{X}}$ which has $\boldsymbol{X}$ as its "boundary process", we shall give an answer to the problem in [6] of disclosing the structure of $\partial M(0)$ as follows (Theorem 6.1): for any $Y \in \partial M(0)$ there exists a local operator $S\left(\frac{1}{i} \frac{d}{d t}\right)$ in the space of Fourier hyperfunctions such that

$$
Y=S\left(\frac{1}{i} \frac{d}{d x}\right) \mu_{X}(0)
$$

Conversely, any $Y \in M$ satisfying (1.17) belongs to $\partial M(0)$.

From $\S 7$ through $\S 9$, we shall suppose that $\boldsymbol{X}$ has the Markovian property and that the representation kernel $\sqrt{2 \pi}^{-1} E$ in (1.2) is real analytic in $(0, \infty)$.

In $\S 7$ we shall first prove that $E$ is real analytic in $(0, \infty)$ if and only if the local operator $P\left(\frac{1}{i} \frac{d}{d x}\right)$ induced by $P(\cdot)$ in (1.14) is elliptic in the sense of hyperfunctions (Theorem 7.1). Considering the problem of prediction by means of a bounded segment of the past, H. Dym and H. P. McKean, Jr. [6] have reduced it to the problem of prediction by means of the germ field. The next aim in this section is to solve its problem by introducing a prediction operator using the germ field (Theorem 7.2, Remark 7.3). 
In $\S 8$ we shall obtain a formula for $E$ as an application of Theorem 7.1 (Theorem 8.1). Investigating this formula, we shall show that $\sqrt{2 \pi}{ }^{-1} E$ might be called a Goursat kernel in a certain modified sense (Theorem 8.2).

In $\S 9$, supposing that $P(\cdot)$ in (1.14) is a transcendental function with an infinite number of roots, we shall introduce a stochastic process $\mathscr{Y}=$ $(\mathscr{Y}(t) ; t \in \boldsymbol{R})$ whose state space is an infinite dimensional Euclidean space $\boldsymbol{R}^{\infty}$. Denoting by $y_{n}(t)\left(n \in N_{*}\right)$ the components of $\mathscr{Y}(t)$, we shall prove that, for almost all $\omega \in \Omega, y_{0}(t)$ is continuous but not differentiable in $t$ and each $y_{n}(t)(n \in N)$ is continuously differentiable in $t$. Furthermore we shall show that for any $t \in R$ a set of random variables $\left\{y_{n}(t) ; n \in N_{*}\right\}$ is linearly independent in the space $\boldsymbol{M}$ and that the process $\boldsymbol{X}$ is the same as the process $\mathscr{Y}$ as far as the Borel fields are concerned (Theorems 9.1 and 9.2).

\section{Acknowledgement}

It is my pleasure to express my sincere gratitude to Professor N. Ikeda for his valuable advices and hearty encouragement.

\section{§ 2. Operator $K$}

Throughout this paper we shall consider a real-valued stationary Gaussian process $X=(X(t) ; t \in R)$ on a probability space $(\Omega, F, P)$ with zero mean and continuous correlation function $k$ :

$$
k(s-t)=E(X(s) X(t)), \quad s, t \in \boldsymbol{R} .
$$

For each open set $D$ in $\boldsymbol{R}$, we define a sub- $\sigma$-field $\boldsymbol{F}(D)$ of $\boldsymbol{F}$ by (2.2) $\boldsymbol{F}(D)=$ the smallest $\sigma$-field for which $X(t)(t \in D)$ is measurable.

Furthermore we set $\boldsymbol{F}^{-}(D)=\bigcap_{\varepsilon>0} F\left(D_{\varepsilon}\right)$, where $D_{\varepsilon}$ means an $\varepsilon$-neighbourhood of $D$. We then introduce four sub- $\sigma$-fields of $\boldsymbol{F}$ which will be analysed in this paper.

Definition 2.1. For any $a \in \boldsymbol{R}$ we set

$$
\partial \boldsymbol{F}(a)=\bigcap_{\varepsilon>0} \boldsymbol{F}((a-\varepsilon, a+\varepsilon)), \boldsymbol{F}^{+}(a)=\boldsymbol{F}((a, \infty)), \boldsymbol{F}^{-}(a)=\boldsymbol{F}((-\infty, a))
$$

and

$\boldsymbol{F}^{+/-}(a)=$ the smallest sub- $\sigma$-field of $\boldsymbol{F}^{-}(a)$ under whose knowledge $\boldsymbol{F}^{+}(a)$ is independent of $\boldsymbol{F}^{-}(a)$. (Such a field always exists and is called the smallest splitting field of $\boldsymbol{F}^{+}(\alpha)$ upon $\boldsymbol{F}^{-}(\alpha)([15])$.)

Now, following K. Urbanik [26] and N. Levinson-H. P. McKean, Jr. 
[14], we give the definition of Markovian property which is a principal subject in this paper.

DEFINITION 2.2. We say that $\boldsymbol{X}$ has the Markovian property if

$$
\partial \boldsymbol{F}(0)=\boldsymbol{F}^{+/-}(0) .
$$

REMARK 2.1. Since $P\left(A \mid \boldsymbol{F}^{-}(a)\right)=P\left(A \mid \boldsymbol{F}^{+/-}(a)\right)$ for any $a \in \boldsymbol{R}$ and any $A \in F^{+}(a)$, it follows from Lemma 2.1 (i) (iii), Corollary 2.1 and Lemma 2.6 (ii) that for any $a \in R$

$$
\partial \boldsymbol{F}(a) \subset \boldsymbol{F}^{+}(a) \cap \boldsymbol{F}^{-}(a) \subset \boldsymbol{F}^{+/-}(a) \subset \boldsymbol{F}^{-}(a) .
$$

Noting this, we see that Definition 2.2 is equivalent to the one in $\S 1$.

Let $M$ be a real Hilbert subspace of $L^{2}(\Omega, F, P)$ obtained by closing the linear hull of $\{X(t) ; t \in \boldsymbol{R}\}$ with the norm $\|Y\|_{M}=\left(E Y^{2}\right)^{1 / 2}$. Similarly as in (2.2), we define for any open set $D$ in $\boldsymbol{R}$ a closed subspace $\boldsymbol{M}(D)$ of $\boldsymbol{M}$ by

$$
M(D)=\text { the closed linear hull of }\{X(t) ; t \in D\} .
$$

Moreover we set $\boldsymbol{M}^{-}(D)=\bigcap_{c>0} \boldsymbol{M}\left(D_{\varepsilon}\right)$.

Definition 2.3. (i) For any $a \in \boldsymbol{R}$ we put

$$
\partial \boldsymbol{M}(a)=\bigcap_{\bullet>0} \boldsymbol{M}((a-\varepsilon, a+\varepsilon)), \boldsymbol{M}^{+}(a)=\boldsymbol{M}((a, \infty)) \quad \text { and } \quad \boldsymbol{M}^{-}(a)=\boldsymbol{M}((-\infty, a)) .
$$

(ii) For any $a \in \boldsymbol{R}$, we denote by $\boldsymbol{M}^{+/-}(a)$ the projection of $\boldsymbol{M}^{+}(a)$ upon $M^{-}(a)$.

REMARK 2.2. $\boldsymbol{M}^{+/-}(a)$ cannot be always closed.

Then, since $\boldsymbol{X}$ is a Gaussian process, the following Lemma 2.1 can be proved ([15], [25]).

LEMMA 2.1. ( i ) For any open set $D$ in $\boldsymbol{R}, \boldsymbol{F}(D)=\sigma(\boldsymbol{M}(D))$ and $\boldsymbol{F}^{-}(D)=$ $\sigma\left(\boldsymbol{M}^{-}(D)\right) . \quad$ In particular, for any $a \in \boldsymbol{R}, \partial \boldsymbol{F}(a)=\sigma(\partial \boldsymbol{M}(a)), \boldsymbol{F}^{+}(a)=\sigma\left(\boldsymbol{M}^{+}(a)\right)$ and $\boldsymbol{F}^{-}(a)=\sigma\left(\boldsymbol{M}^{-}(a)\right)$.

(ii) For any open set $D$ in $\boldsymbol{R}, \boldsymbol{M}(D)$ and $\boldsymbol{M}^{-}(D)$ coincide with the spaces of those elements of $\boldsymbol{M}$ which are $\boldsymbol{F}(D)$-measurable and $\boldsymbol{F}^{-}(D)$-measurable, respectively. Especially, for any $a \in \boldsymbol{R}, \partial \boldsymbol{M}(a), \boldsymbol{M}^{+}(a)$ and $\boldsymbol{M}^{-}(a)$ accord with the spaces of those elements of $\boldsymbol{M}$ which are $\partial \boldsymbol{F}(a)$-measurable, $\boldsymbol{F}^{+}(a)$ measurable and $\boldsymbol{F}^{-}(a)$-measurable, respectively.

(iii) For any $a \in \boldsymbol{R}, \boldsymbol{F}^{+/-}(a)=\sigma\left(\boldsymbol{M}^{+/-}(a)\right)$.

REMARK 2.3. By Remark 2.2, $M^{+/-}(a)$ does not always equal the closed subspace of those elements of $\boldsymbol{M}$ which are $\boldsymbol{F}^{+/-}(a)$-measurable.

We denote by $\boldsymbol{K}$ the real Hilbert space with $k$ in (2.1) reproducing kernel and by $(,)_{K}$ its inner product. This is possible because $k$ is non-negative definite ([2]). From the general theory of such a space, we see that our 
space $K$ is included in the space of bounded continuous functions, contains $k(\cdot-t)(t \in \boldsymbol{R})$ as its elements and satisfies the following properties :

$\boldsymbol{K}=$ the closed linear hull of $\{k(\cdot-t) ; t \in \boldsymbol{R}\}$;

$$
(u, k(\cdot-t))_{\boldsymbol{K}}=u(t), \quad \text { for any } u \in \boldsymbol{K} \text { and any } t \in \boldsymbol{R} .
$$

Similarly as in (2.4), we define for any open set $D$ in $R$ a closed subspace $\boldsymbol{K}(D)$ of $\boldsymbol{K}$ by

(2.7) $K(D)=$ the closed linear hull of $\{k(\cdot-t) ; t \in D\}$.

Furthermore we set $K^{-}(D)=\bigcap_{c>0} K\left(D_{\varepsilon}\right)$.

DEFINITION 2.4. (i) For any $a, a_{1}, a_{2}, \cdots, a_{n} \in \boldsymbol{R}$, we put

$$
\begin{aligned}
& \partial K\left(a_{1}, \cdots, a_{n}\right)=\bigcap_{\varepsilon>0} K\left(\bigcup_{j=1}^{n}\left(a_{j}-\varepsilon, a_{j}+\varepsilon\right)\right) \\
& K^{+}(a)=K((a, \infty)) \text { and } K^{-}(a)=K((-\infty, a))
\end{aligned}
$$

(ii) For any $a \in \boldsymbol{R}$, we denote by $\boldsymbol{K}^{+/-}(a)$ the projection of $\boldsymbol{K}^{+}(a)$ upon $\boldsymbol{K}^{-}(a)$.

REMARK 2.4. $K^{+/-}(a)$ cannot be always closed.

By (2.1) and (2.6), $\left\|\sum_{j=1}^{n} c_{j} k\left(\cdot-t_{j}\right)\right\|_{K}=\left\|\sum_{j=1}^{n} c_{j} X\left(t_{j}\right)\right\|_{M}$. Therefore, we have

Proposition 2.1. There uniquely exists a unitary operator $U_{1}$ from $\boldsymbol{K}$ onto $\boldsymbol{M}$ such that $U_{1}(k(\cdot-t))=X(t)$, for any $t \in \boldsymbol{R}$.

Immediately from (2.4), (2.7) and Proposition 2.1, we get

CoRollary 2.1. Under the operator $U_{1}$, for any open set $D$ in $\boldsymbol{R}, \boldsymbol{K}(D)$ and $\boldsymbol{K}^{-}(D)$ correspond to $\boldsymbol{M}(D)$ and $\boldsymbol{M}^{-}(D)$, respectively. Particularly, for any $a \in \boldsymbol{R}, \partial \boldsymbol{K}(a), \boldsymbol{K}^{+}(a), \boldsymbol{K}^{-}(a)$ and $\boldsymbol{K}^{+/-}(a)$ correspond to $\partial \boldsymbol{M}(a), \boldsymbol{M}^{+}(a), \boldsymbol{M}^{-}(a)$ and $M^{+/-}(a)$, respectively.

In the following we take our study on the space $K$ rather than $M$ from an operator-theoretical point of view. For any open set $D$ in $R$, we define a closed subspace $K_{0}(D)$ of $K$ by

$$
\boldsymbol{K}_{0}(D)=\{u \in \boldsymbol{K} ; u=0 \quad \text { in } D\} .
$$

We note that the closedness of $K_{0}(D)$ is proved by (2.6). Furthermore, using (2.6) and (2.7), we have

Lemma 2.2. For any open set $D$ in $\boldsymbol{R}$,

(i) $\boldsymbol{K}(D)=\boldsymbol{K}_{0}(D)^{\perp}$ (the orthogonal complement of $\boldsymbol{K}_{0}(D)$ ),

(ii) the linear hull of $\bigcup_{n=1}^{\infty} K\left(D_{n}\right)$ is dense in $K(D)$ for any family $\left(D_{n}\right)_{n=1}^{\infty}$ of open sets in $D$ such that $\bigcup_{n=1}^{\infty} D_{n}$ is dense in $D$. 
Noting (2.6) and (2.7), it is easy to see

LEMMA 2.3. For any $u \in K$ and any $a, b, t \in \boldsymbol{R}, a<b$,

(i ) $\check{u} \in \boldsymbol{K}, \quad\|u\|_{K}=\|\check{u}\|_{K}$,

(ii) $u(\cdot+t) \in \boldsymbol{K}, \quad\|u\|_{\boldsymbol{K}}=\|u(\cdot+t)\|_{\boldsymbol{K}}$,

(iii) $\lim _{: \downarrow 0} u(\cdot \pm \varepsilon)=u$ in $K$,

(iv) if $u \in \boldsymbol{K}((a, b)), \partial \boldsymbol{K}(a), \boldsymbol{K}^{+}(a)$ or $\boldsymbol{K}^{-}(a)$, then $u(\cdot+t) \in \boldsymbol{K}((a-t, b-t))$, $\partial \boldsymbol{K}(a-t), \boldsymbol{K}^{+}(a-t)$ or $\boldsymbol{K}^{-}(a-t)$, respectively.

Immediately from Lemma 2.3, we get

LEMMA 2.4. If $\partial \boldsymbol{K}(0)=\boldsymbol{K}^{+/-}(0)$, then

$$
\partial \boldsymbol{K}(\alpha)=\boldsymbol{K}^{+/-}(a), \quad \text { for any } a \in \boldsymbol{R} .
$$

By (2.7) and Lemma 2.3, we obtain

LEMMA 2.5. (i ) $K\left(D_{1}\right) \subset K\left(D_{2}\right)$, for any open sets $D_{1}, D_{2}$ in $R, D_{1} \subset D_{2}$, (ii) $\boldsymbol{K}^{+}(a) \supset \boldsymbol{K}^{+}(b)$ and $\boldsymbol{K}^{-}(a) \subset \boldsymbol{K}^{-}(b)$, for any $a, b \in \boldsymbol{R}, a<b$,

(iii) $\boldsymbol{K}^{+}(a)=\bigcap_{\varepsilon>0} \boldsymbol{K}^{+}(a-\varepsilon)$ and $\boldsymbol{K}^{-}(a)=\bigcap_{c>0} \boldsymbol{K}^{-}(a+\varepsilon)$, for any $a \in \boldsymbol{R}$.

Using Lemmas 2.3 and 2.5, we can show

LEMMA 2.6. (i) $\partial K(a)=\bigcap_{\varepsilon>0} K((a, a+\varepsilon))=\bigcap_{\varepsilon>0} K((a-\varepsilon, a))$, for any $a \in \boldsymbol{R}$,

(ii) $\quad \partial K(a) \subset K^{+}(a) \cap K^{-}(a) \subset K^{+/-}(a) \subset K^{-}(a)$, for any $a \in R$.

Though $\boldsymbol{M}^{+/-}(0)$ and $\boldsymbol{K}^{+/-}(0)$ cannot be always closed as noted in Remarks 2.2 and 2.4, the next Proposition 2.2 shows that if $X$ has the Markovian property, then they are closed in $M$ and $K$, respectively.

Proposition 2.2. The following (i), (ii) and (iii) are equivalent:

(i) $\boldsymbol{X}$ has the Markovian property;

(ii) $\partial M(0)=M^{+/-}(0)$;

(iii) $\partial K(0)=K^{+/-}(0)$.

Proof. By Corollary 2.1, it is clear that (ii) is equivalent to (iii). Furthermore, by Lemma 2.1 (i) (iii), (ii) implies (i). Conversely, suppose that (i) holds and fix any $Y \in \boldsymbol{M}^{+/-}(0)$. Then, since $Y$ is $\boldsymbol{F}^{+/-}(0)$-measurable by Lemma 2.1 (iii), it is $\partial F(0)$-measurable. Therefore, it follows from Lemma 2.1 (ii) that $Y \in \partial M(0)$ and hence $M^{+/-}(0) \subset \partial M(0)$. Thus, we have (ii) because the converse inclusion always holds by Corollary 2.1 and Lemma 2.6 (ii).

Combining Lemmas 2.5 and 2.6, we get

LEMMA 2.7. For any $a, b \in \boldsymbol{R}, a<b$,

(i) $\quad \partial \boldsymbol{K}(a) \cup \partial \boldsymbol{K}(b) \subset \boldsymbol{K}((a, b)) \subset \boldsymbol{K}^{-}((a, b)) \subset \boldsymbol{K}^{+}(a) \cap \boldsymbol{K}^{-}(b)$,

(ii) $\quad \partial \boldsymbol{K}(a, b) \subset \boldsymbol{K}^{-}((a, b)) \cap \boldsymbol{K}^{-}((-\infty, a) \cup(b, \infty))$.

By Proposition 2.2, the next Lemma 2.8 shows that the Markovian property is a dual concept.

LEMMA 2.8. 'For any $a \in \boldsymbol{R}$, the following conditions are equivalent: 
( i ) $\partial \boldsymbol{K}(a)=\boldsymbol{K}^{+/-}(a)$;

(ii) $K^{-}(a)=K^{+}(a)^{\perp} \oplus \partial K(a)$;

(iii) $\partial \boldsymbol{K}(a)=\boldsymbol{K}^{-/+}(a)$ (三the projection of $\boldsymbol{K}^{-}(a)$ upon $\left.\boldsymbol{K}^{+}(a)\right)$;

(iv) $\boldsymbol{K}^{+}(a)=\boldsymbol{K}^{-}(a)^{\perp} \oplus \partial \boldsymbol{K}(a)$.

Proof. Suppose that (i) holds. By Lemma 2.6 (ii), $\partial K(a)$ is orthogonal to $\boldsymbol{K}^{+}(a)^{\perp}$ and it is contained in $\boldsymbol{K}^{-}(a)$. Fix any $u \in \boldsymbol{K}^{+}(a)^{\perp}$. Since $\boldsymbol{K}^{+/-}(a)$ $\subset K^{+}(a)$ by our assumption (i) and Lemma 2.6 (ii), we see that for any $v \in K^{+}(a) \quad\left(P_{K^{-(a)}} u, v\right)_{K}=(u, v)_{K^{-}}\left(u, P_{K^{-(a)}} v\right)_{K}=0 .{ }^{8)} \quad$ This implies that $u \in K^{-}(a)$ by Lemma 2.2 (ii) and so $K^{+}(a)^{\perp} \oplus \partial K(a) \subset K^{-}(a)$. Next, fix any $u \in K^{-}(a)$. Since $P_{K^{+}(a)+} u=u-P_{K^{+}(a)+} u \in K^{-}(a)$, it follows from (i) and Lemma 2.6 (ii) that $P_{K^{+(a)}} u \in \partial K(a)$. Hence, we obtain (ii). Conversely, suppose that (ii) holds and fix any $u \in K^{+}(a)$. Then, since $K^{+}(a)^{\perp} \subset K^{-}(a)$, we see that for any $v \in K^{+}(a)^{\perp}\left(P_{K^{-(a)}} u, v\right)_{K}=\left(u, P_{K^{-(a)}} v\right)_{K}=(u, v)_{K}=0$. This shows that $P_{K-(a)} u \in K^{+}(a)$ and so it belongs to $\partial K(a)$ by (ii) and Lemma 2.6 (ii). Thus, we have (i). Similarly, the equivalence of (iii) and (iv) is proved. Finally, by Lemma 2.6 (ii), (i) and (iii) follow from (iv) and (ii), respectively.

In connection with the problem of extrapolation ([6], [27]), we shall show

Proposition 2.3. If $\partial K(0)=K^{+/-}(0)$, then for any $a, b \in \boldsymbol{R}, a<b, \partial K(a, b)$ $=P_{K-((a, b))} K^{-}((-\infty, a) \cup(b, \infty))$.

Proof. By Lemma 2.7 (ii), $\partial K^{(}(a, b) \subset P_{K^{-((a, b))}} K^{-}((-\infty, a) \cup(b, \infty))$. Fix any $u \in K^{-}((-\infty, a) \cup(b, \infty))$ and take any $\varepsilon \in\left(0, \frac{1}{2}(b-a)\right)$. Then, noting $u \in K((-\infty, a+\varepsilon) \cup(b-\varepsilon, \infty))$, and using Lemma 2.4, we can prove that $P_{K((a-\delta, b+\delta))} u \in K((a-\varepsilon, a+\varepsilon) \cup(b-\varepsilon, b+\varepsilon))$ for any $\delta \in(0, \varepsilon)$. Therefore, since $P_{K-((a, b))} u=\lim _{\delta \downarrow 0} P_{K((a-\delta, b+\delta))} u$, we see that $P_{K-((a, b))} u \in K((a-\varepsilon, a+\varepsilon) \cup$ $(b-\varepsilon, b+\varepsilon))$ and so it belongs to $\partial K(a, b)$ because $\varepsilon$ is arbitrary. Thus, we have proved Proposition 2.3.

(Q.E.D.)

It will be found later that the next Proposition 2.4 yields that the technical condition in [6] (see (2.28)) holds if $\boldsymbol{X}$ has the Markovian property.

Proposition 2.4. If $\partial K(0)=K^{+/-}(0)$, then

$$
\boldsymbol{K}((a, b))=\boldsymbol{K}^{-}((a, b)), \quad \text { for any } a, b \in \boldsymbol{R}, a<b .
$$

Proof. By the same argument as in Proposition 2.3, we see that $P_{K^{-(a, b)}} K^{-}(a) \subset \partial K(a)$ and $P_{K^{-((a, b))}} K^{+}(b) \subset \partial K(b)$. Therefore, by Lemma 2.7, $P_{K^{-((a, b))}}\left(K^{-}(a) \cup K((a, b)) \cup K^{+}(b)\right) \subset K((a, b))$. Noting Lemma 2.2 (ii), this completes the proof of Proposition 2.4.

It will be shown in $\S 5$ that the next Lemma 2.9 (iii) is equivalent to

8) For any closed subspace $\boldsymbol{M}_{1}$ of $\boldsymbol{M}$, we denote by $\boldsymbol{P}_{\boldsymbol{M}_{1}}$ the projection operator on $M_{1}$. 
the Markovian property of $\boldsymbol{X}$ in case $\boldsymbol{X}$ is purely non-deterministic.

LEMMA 2.9. If $\partial \boldsymbol{K}(0)=\boldsymbol{K}^{+/-}(0)$, then, for any $a, b \in \boldsymbol{R}, a<b$,

(i ) $\boldsymbol{K}((a, b))=\boldsymbol{K}^{+}(a) \cap \boldsymbol{K}^{-}(b)=\boldsymbol{P}_{\boldsymbol{K}^{-}\left({ }^{\prime}\right)} \boldsymbol{K}^{+}(a)$,

(ii) $P_{K-(b)} K_{0}((-\infty, a)) \subset K(a, b)$,

(iii) $\boldsymbol{K}_{0}((a, b)) \subset \boldsymbol{K}^{-}(a) \oplus \boldsymbol{K}^{-}(b)^{\perp}$.

Proof. By Lemmas 2.4 and 2.7, $P_{\boldsymbol{K}^{-(b)}}\left(\boldsymbol{K}((\alpha, b)) \cup \boldsymbol{K}^{+}(b)\right) \subset \boldsymbol{K}((a, b))$. Since $\boldsymbol{K}^{+}(a)=$ the closed linear hull of $\boldsymbol{K}((a, b)) \cup \boldsymbol{K}^{+}(b)$, this implies that $P_{K^{-(b)}} K^{+}(a) \subset K((a, b))$. Therefore, (i) follows from Lemma 2.7 (i). Furthermore, since Lemmas 2.2 (i), 2.4 and 2.8 (iv) imply that $K_{0}((-\infty, a)) \subset K^{+}(a)$, (ii) follows from (i). Finally, since $\left(\boldsymbol{K}^{-}(a) \oplus \boldsymbol{K}^{-}(b)^{\perp}\right)^{\perp}=\boldsymbol{K}_{0}((-\infty, a)) \cap \boldsymbol{K}^{-}(b)$ by Lemma 2.2 (i), (iii) follows from (ii) and Lemma 2.2 (i).

Our next aim is to introduce an operator from $K$ into some function space and then to rephrase Lemma 2.9 (iii) by the local property of the operator. Now, from Wold's decomposition theorem ([11]), there exist two stationary Gaussian processes $\boldsymbol{X}_{j}=\left(X_{j}(t) ; t \in \boldsymbol{R}\right)(j=1,2)$ with the same property as $\boldsymbol{X}$ such that $X(t)=X_{1}(t)+X_{2}(t)$ for any $t \in \boldsymbol{R}$. Moreover we know that $\boldsymbol{X}_{1}$ and $\boldsymbol{X}_{2}$ are orthogonal in $\boldsymbol{M}$ and $\boldsymbol{X}_{1}$ is deterministic, $\boldsymbol{X}_{2}$ is purely non-deterministic :

$$
\bigcap_{a \in R} M_{\bar{X}_{1}}(a)=M_{X_{1}} \text { and } \bigcap_{a \in R} M_{\bar{X}_{2}}(\alpha)=\{0\} .
$$

Then, it can be seen that $\boldsymbol{X}$ has the Markovian property if and only if both $\boldsymbol{X}_{1}$ and $\boldsymbol{X}_{2}$ have the Markovian property. For a while, we consider the case $\boldsymbol{X}$ is deterministic. In this case, Lemma 2.9 (i) is a mere paraphrase of the Markovian property and Lemma 2.9 (ii) (iii) always holds by Corollary 2.1 and Lemma 2.2 (i). However, it is easy to verify that $\boldsymbol{X}$ has the Markovian property if and only if $\partial K(a)=K$ for any $a \in R$. This is equivalent to the fact that each element of $K$ has the property of quasi-analyticity, namely if $u \in K$ vanishes in a neighbourhood of some point, then $u=0$. For example, we see that if $k$ is real analytic, then $\boldsymbol{X}$ is deterministic and has the Markovian property.

From now till last in this parer, we suppose that $\boldsymbol{X}$ is purely nondeterministic. Then, it is well-known ([11]) that the spectral measure of $k$ has a Hardy density $\Delta:$ that is,

$$
\begin{gathered}
k(t)=\int_{\boldsymbol{R}} e^{-i t \lambda} \Delta(\lambda) d \lambda \equiv \hat{\Delta}(t), \quad t \in \boldsymbol{R}, \\
\int_{\boldsymbol{R}} \frac{\lg \Delta(\lambda)}{1+\lambda^{2}} d \lambda>-\infty
\end{gathered}
$$

We denote by $h$ the outer part of $\Delta([9])$ : 


$$
h(z)=\exp \left\{\frac{1}{2 \pi i} \int_{R} \frac{1+z \lambda}{\lambda-z} \frac{\lg \Delta(\lambda)}{1+\lambda^{2}} d \lambda\right\}, \quad z \in C^{+9}{ }^{9)}
$$

This is possible by (2.10). Then, since $h$ satisfies the condition

$$
h \in \mathcal{O}\left(C^{+}\right)^{10)} \text { and } \sup _{y>0} \int_{R}|h(x+i y)|^{2} d x<\infty,
$$

it can be proved ([9]) that, for almost all $\lambda \in \boldsymbol{R}, \underset{\varepsilon \downarrow 0}{\operatorname{li} . \mathrm{m} .} h(\lambda+i \varepsilon) \equiv h(\lambda)$ exists and $h \equiv h(\lambda)$ has the next property:

$$
h \in L^{2}, \overline{h(\lambda)}=h(-\lambda) \quad \text { and } \quad|h(\lambda)|^{2}=\Delta(\lambda), \quad \lambda \in \boldsymbol{R} .
$$

From (2.10) and (2.13), we see that

$$
h(\lambda) \neq 0, \quad \text { for almost all } \lambda \in \boldsymbol{R} .
$$

Next we denote by $E$ the Fourier transform of $h$ :

$$
E(t)=\int_{\boldsymbol{R}} e^{-i t \lambda} h(\lambda) d \lambda, \quad t \in \boldsymbol{R} .
$$

Then, by (2.12), it can be shown ([9)] that

$$
E=0 \quad \text { in }(-\infty, 0) \text {. }
$$

By (2.9), (2.13) and (2.15), we have

$$
\begin{gathered}
E \in L^{2}(\boldsymbol{R}), \\
k=\frac{1}{2 \pi} E * \check{E}=\frac{1}{2 \pi} \check{E} * E .
\end{gathered}
$$

We define a subspace $H^{2+}$ of $L^{2}$ by

$$
H^{2+}=\left\{f \in L^{2} ; \hat{f}=0 \quad \text { in } \quad(-\infty, 0)\right\} .
$$

The following Lemma 2.10 is due to K. Karhunen [11] which plays an important role in this paper.

LEMMA 2.10. For any fixed $f \in H^{2+}$, if for any $t \in(0, \infty)$

$$
\int_{0}^{\infty} E(s) \overline{\hat{f}(t+s)} d s=0, \text { then } f=0 .
$$

We note that Lemma 2.10 is equivalent to

9) $C^{+}=\{z \in C ; \operatorname{Im} z>0\}$.

10) For any open set $D$ in $C$, we denote by $\mathcal{O}(D)$ the set of all holomorphic functions in $D$. 


$$
H^{2+}=\text { the closed linear hull of }\left\{e^{i t \cdot h} ; t>0\right\} \text { in } L^{2} \text {. }
$$

By (2.14) and (2.15), we get

LEMMA 2.11. $\quad L^{2}(\boldsymbol{R})=$ the closed linear hull of $\{E(t-\cdot) ; t \in \boldsymbol{R}\}$.

The following Proposition 2.5 gives a realization of $K$. It will be found in Theorem 2.1 that the operator $K$ is our desirable one.

Proposition 2.5. There uniquely exists a unitary operator $K$ from $K$ onto $L^{2}(\boldsymbol{R})$ such that for any $t \in \boldsymbol{R} K(k(\cdot-t))=\frac{1}{\sqrt{2 \pi}} E(t-\cdot)$. Furthermore, the next relation holds:

$$
u=\frac{1}{\sqrt{2 \pi}} E * K u, \quad \text { for any } u \in K .
$$

Proof. By (2.6) and (2.18),

$$
\left\|\sum_{j=1}^{n} c_{j} k\left(\cdot-t_{j}\right)\right\|_{K}^{2}=\frac{1}{2 \pi} \sum_{i, j=1}^{n} c_{i} c_{j} E * \check{E}\left(t_{i}-t_{j}\right)=\left\|\frac{1}{\sqrt{2 \pi}} \sum_{j=1}^{n} c_{j} E\left(t_{j}-\cdot\right)\right\|_{L^{2}(\boldsymbol{R})}^{2} \cdot
$$

Therefore, noting (2.5) and Lemma 2.11, we obtain the first part in Proposition 2.5. (2.21) follows from (2.5), (2.18) and the first part if we note that for any $t \in R k(\cdot-t)=\frac{1}{\sqrt{2 \pi}} E *(K(k(\cdot-t)))$.

Similarly, we have

Proposition 2.6. There uniquely exists a unitary operator $\check{K}$ from $\boldsymbol{K}$ onto $L^{2}(\boldsymbol{R})$ such that for any $t \in \boldsymbol{R} \check{K}(k(\cdot-t))=\frac{1}{\sqrt{2 \pi}} E(\cdot-t)$. Moreover, the next relation holds :

$$
u=\frac{1}{\sqrt{2 \pi}} \check{E} * \check{K} u, \quad \text { for any } u \in K
$$

Next we shall characterize the spaces $K^{+}(a)$ and $\boldsymbol{K}^{-}(a)$ by means of two operators $K$ and $\check{K}$.

LEMMA 2.12. (i) $K_{0}((-\infty, a))=\{u \in K ; K u=0$ in $(-\infty, a)\}$, for any $a \in \boldsymbol{R}$,

(ii) $\boldsymbol{K}^{-}(a)=\{u \in \boldsymbol{K} ; K u=0$ in $(a, \infty)\}$, for any $a \in \boldsymbol{R}$.

Proof. Fix any $u \in K_{0}((-\infty, a))$. We set $f=\chi_{(-\infty, 0)}^{(\cdot)} K u(\cdot+a)^{11)}$ and define $g \in H^{2+}$ by $g=\widetilde{f}$. By (2.16) and (2.21), for any $t \in(0, \infty), \int_{0}^{\infty} E(s) \overline{\hat{g}(t+s)} d s=$ $E * K u(a-t)=\sqrt{2 \pi} u(a-t)=0$. Therefore, it follows from Lemma 2.10 that $g=0$ and so $K u=0$ in $(-\infty, a)$. Conversely, let $u$ be any element of $K$ such

11) For any set $B$ in $R \chi_{B}$ denotes the indicator function of $B$. 
that $K u=0$ in $(-\infty, a)$. Then, by (2.16) and (2.21), for any $t \in(-\infty, a)$, $u(t)=\frac{1}{\sqrt{2 \pi}} \int_{a}^{\infty} E(t-s) K u(s)^{\prime} d s=0 . \quad$ Hence, we have proved (i). (ii) follows from (i), Lemma 2.2 (i) and Proposition 2.5.

(Q.E.D.)

By Lemmas 2.3 (i), 2.12 and Propositions 2.5, 2.6, we get

Lemma 2.13. (i ) $\boldsymbol{K}_{0}((a, \infty))=\{u \in \boldsymbol{K} ; \check{K} u=0$ in $(a, \infty)\}$, for any $a \in \boldsymbol{R}$, (ii) $\boldsymbol{K}^{+}(a)=\{u \in \boldsymbol{K} ; \check{K} u=0$ in $(-\infty, a)\}$, for any $a \in \boldsymbol{R}$.

Furthermore we shall show

LEMMA 2.14. For any $a, b \in \boldsymbol{R}, a<b$,

$$
\boldsymbol{K}^{-}(a) \oplus \boldsymbol{K}^{-}(b)^{\perp}=\{u \in \boldsymbol{K} ; K u=0 \text { in }(a, b)\} .
$$

Proof. Using Lemmas 2.2 (i), 2.12 and Proposition 2.5, $\{u \in K ; K u=0$ in $(a, b)\}^{\perp}=\{u \in K ; K u=0 \quad$ in $(-\infty, a) \cup(b, \infty)\}=K^{-}(a)^{\perp} \cap K^{-}(b)$. Noting Lemma 2.5 (ii), this completes the proof of Lemma 2.14.

(Q.E.D.)

Now, after above preparations, we are in a position to prove main Theorem 2.1 in this section. For this, we give the definition of local property of $K$.

DEFinition 2.5. We say that the operator $K$ has the local property if $u \in K_{0}((a, b))$, then $K u=0$ in $(a, b)(-\infty<a<b<\infty)$.

THEOREM 2.1. If the process $\boldsymbol{X}$ has the Markovian property, then the operator $K$ has the local property.

Proof. Fix any $u \in K_{0}((a, b))$. Then, by Proposition 2.2 and Lemma 2.9 (iii), $u \in K^{-}(a) \oplus K^{-}(b)^{\perp}$. This implies that $K u=0$ in $(a, b)$ by Lemma 2.14.

Finally, for later use, we shall examine the relation between the spaces $\boldsymbol{K}$ and $\boldsymbol{Z}_{\Delta}$, where $\boldsymbol{Z}_{\Delta}$ is a real Hilbert space defined by $\boldsymbol{Z}_{\Delta}=\left\{\varphi \in L^{2}(\boldsymbol{R} \rightarrow \boldsymbol{C} ; \Delta d \lambda)\right.$; $\overline{\varphi(\lambda)}=\varphi(-\lambda)\}$ and it is used in [6], [14] and [26] as stated in $\S 1$. We note that for any fixed $\varphi \in Z_{\Delta}$

$$
\begin{gathered}
\varphi \Delta \in L^{1}, \\
\text { if } \widehat{\varphi}=0, \quad \text { then } \varphi=0 \text {. }
\end{gathered}
$$

Similarly as in (2.7), we define for any open set $D$ in $\boldsymbol{R}$ a closed subspace $Z_{4}(D)$ of $Z_{\Delta}$ by

$$
Z_{\Delta}(D)=\text { the closed linear hull of }\left\{e^{i t} \cdot t \in D\right\} .
$$

Moreover we set $Z_{4}^{-}(D)=\bigcap_{s>0} Z_{4}\left(D_{s}\right) . \quad$ By (2.24) and (2.25),

$$
Z_{\Delta}=Z_{\Delta}(R)
$$

Definition 2.6. (i) For any $a, a_{1}, \cdots, a_{n} \in \boldsymbol{R}$, we put 


$$
\begin{aligned}
& \partial Z_{\Delta}\left(a_{1}, \cdots, a_{n}\right)=\bigcap_{\varepsilon>0} Z_{\Delta}\left(\bigcup_{j=1}^{n}\left(a_{j}-\varepsilon, a_{j}+\varepsilon\right)\right), \quad Z_{\Delta}^{+}(a)=Z_{\Delta}((a, \infty)) \\
& \text { and } Z_{\Delta}^{-}(a)=Z_{\Delta}((-\infty, a)) .
\end{aligned}
$$

(ii) For any $\alpha \in \boldsymbol{R}$, we denote by $\boldsymbol{Z}_{\Delta}^{+/-}(\alpha)$ the projection of $\boldsymbol{Z}_{\Delta}^{+}(\alpha)$ upon $Z_{\overline{4}}^{-}(a)$.

REMARK 2.5. $Z_{\Delta}^{+/-}(a)$ cannot be always closed.

Proposition 2.7. There uniquely exists a unitary operator $U_{2}$ from $K$ onto $Z_{\Delta}$ such that for any $t \in \boldsymbol{R} U_{2}(k(\cdot-t))=e^{i t}$. Furthermore, the next relation holds :

$$
u=\widehat{\left(U_{2} u\right) \Delta}, \quad \text { for any } u \in K \text {. }
$$

Proof. By (2.6) and (2.9)

$$
\left\|\sum_{j=1}^{n} c_{j} k\left(\cdot-t_{j}\right)\right\|_{K}^{2}=\left\|\sum_{j=1}^{n} c_{j} e^{i t_{j} \cdot}\right\|_{z_{\Delta}}^{2} .
$$

Therefore, noting (2.5), (2.25) and (2.26), we obtain the first part. (2.27) follows from (2.5), (2.9) and the first part if we note that for any $t \in \boldsymbol{R}$ $k(\cdot-t)=\int_{R} e^{-i \cdot \lambda} U_{2}(k(\cdot-t))(\lambda) \Delta(\lambda) d \lambda$.

Immediately from (2.7), (2.25) and Proposition 2.7, we have

COROLlaRY 2.2. Under the operator $U_{2}$, for any open set $D$ in $R, K(D)$ and $\boldsymbol{K}^{-}(D)$ correspond to $Z_{4}(D)$ and $Z_{4}^{-}(D)$, respectively. Especially, for any $a, a_{1}, \cdots, a_{n} \in \boldsymbol{R}, \quad \partial \boldsymbol{K}\left(a_{1}, \cdots, a_{n}\right), \boldsymbol{K}^{+}(a), \boldsymbol{K}^{-}(a)$ and $\boldsymbol{K}^{+/-}(a)$ correspond to $\partial Z_{\Delta}\left(a_{1}, \cdots, a_{n}\right), Z_{\Delta}^{+}(a), Z_{\Delta}^{-}(a)$ and $Z_{\Delta}^{+/-}(a)$, respectively.

Therefore, combining Proposition 2.2 with Corollary 2.2, we get

Proposition 2.8. The following (i) and (ii) are equivalent:

(i) $\boldsymbol{X}$ has the Markovian property;

(ii) $\partial Z_{\Delta}(0)=Z_{\Delta}^{+/-}(0)$.

REMARK 2.6. By Corollary 2.2, we obtain a statement on $Z_{\Delta}$ corresponding to Lemma 2.6, which has been proved in [14].

REMARK 2.7. As noted in front of Proposition 2.4, it follows from Propositions 2.2, 2.4 and Corollary 2.2 that $X$ having the Markovian property satisfies the next (2.28), which is called a technical condition in [6] :

$Z_{\bar{\Delta}}^{-}((a, b))$ is continuous in the pair $a, b$ in the sense that $\bigcup_{a<c<a<b} Z_{\bar{\Delta}}^{-}((c, d))$ is dense in $Z_{4}^{-}((a, b))$.

In concluding this section, we shall prove

LEMMA 2.15. For any $f \in L^{2}(\boldsymbol{R}), f=\frac{1}{\sqrt{2 \pi}}\left\{\left(U_{2} K^{-1} f\right) \check{h}\right\}^{\wedge}$. 
Proof. It follows from (2.15), (2.21) and (2.27) that $\sqrt{2 \pi}-1 \cdot E * f=$ $\left\{\left(U_{2} K^{-1} f\right) \Delta\right\}^{\wedge}$ and so $\sqrt{2 \pi} h \tilde{f}=\left(U_{2} K^{-1} f\right) \Delta$. Therefore, by (2.13), this completes the proof of Lemma 2.15.

(Q.E.D.)

\section{§3. M. Sato's hyperfunctions}

In this section we shall prepare several definitions and results about M. Sato's hyperfunctions from M. Sato ([18], [19], [20], [21], [22]) and T. Kawai ([12]) which will be used in the sequel.

Setting $\boldsymbol{D}=[-\infty, \infty]$, we define a pre-sheaf $\widetilde{\mathscr{O}}=(\widetilde{\mathscr{O}}(V) ; V$ open in $D \times \sqrt{-1} R$ ) over $D \times \sqrt{-1} R$ as follows :

(3.1) $\tilde{\mathscr{O}}(V)=$ the set of all holomorphic functions $f$ in $V \cap C$ such that for any $\varepsilon>0$ and any compact set $K$ in $V$, the estimate $\sup _{z \in K \cap c}\left|f(z) e^{-\epsilon|z|}\right|$ $<\infty$ holds.

DEFINITION 3.1. This pre-sheaf $\tilde{\mathscr{O}}$ constitutes a sheaf over $D \times \sqrt{-1} R$ and is called the sheaf of slowly increasing holomorphic functions.

For any open set $D$ in $D$, we denote by $\mathscr{V}(D)$ the set of all open sets $V$ in $D \times \sqrt{-1} R$ which contain $D$ as relatively closed set. Take any open set $D$ in $D$ and fix it. In the set of couples $(\varphi, V), \varphi \in \tilde{O}(V-D)$ and $V \in \mathscr{V}(D)$, we introduce the following equivalence relation: two couples $\left(\varphi_{j}, V_{j}\right)(j=1,2)$ are said to be equivalent if and only if

$$
\varphi_{1}(z)=\varphi_{2}(z)+f(z) \quad \text { for any } z \in V-D
$$

with some $f \in \tilde{\mathscr{O}}(V)$ and $V \in \mathscr{V}(D), V \subset V_{1} \cap V_{2}$. We denote by $[\varphi, V]$ the equivalence class determined by a couple $(\varphi, V)$ and say it a Fourier hyperfunction on $D$ defined by $(\varphi, V)$. A couple $(\varphi, V)$ is called a defining function of the Fourier hyperfunction $[\varphi, V]$. We denote by $\mathscr{R}(D)$ the set of all Fourier hyperfunctions on $D$ and in particular, set $\mathscr{B}(D)=\mathscr{R}(D)$ if $D$ is included in $\boldsymbol{R}$.

DEFINITION 3.2. The pre-sheaves $\mathscr{R}=(\mathscr{R}(D) ; D$ open in $D), \mathscr{B}=(\mathscr{B}(D)$; $D$ open in $R$ ) constitute sheaves over $D, R$ and are called the sheaf of Fourier hyperfunctions, the one of hyperfunctions, respectively.

Next we define other pre-sheaf $\mathcal{Q}=(\mathscr{O}(V) ; V$ open in $D \times \sqrt{-1} R)$ over $D \times \sqrt{-1} R$ as follows :

(3.3) $\mathcal{Q}(V)=$ the set of all holomorphic functions $f$ in $V \cap C$ such that for any compact set $K$ in $V$ there exists a constant $\delta_{K}>0$ and the estimate $\sup _{z \in K \cap \boldsymbol{C}}\left|f(z) e^{\delta_{K}|z|}\right|<\infty$ holds. 
DEFINITION 3.3. This pre-sheaf $\mathcal{Q}$ constitutes a sheaf over $D \times \sqrt{-1} R$ and is called the sheaf of rapidly decreasing holomorphic functions.

We define $\mathscr{Q}(\boldsymbol{D})$ by the inductive $\operatorname{limit} \lim \mathcal{O}_{c}^{m}\left(U_{m}\right)$, where for each $m \in N$ $U_{m}=\boldsymbol{D} \times \sqrt{-1}\left(-\frac{1}{m}, \frac{1}{m}\right)$ and $\mathcal{O}_{c}^{m}\left(U_{m}\right)$ is the Banach space of all elements $f \in \mathcal{O}\left(U_{m}\right)$ which are continuous in $\bar{U}_{m} \cap C$ and for which there exists a constant $M>0$ such that $|f(z)| \leqslant M e^{-1 / m|z|}$ for any $z \in U_{m} \cap C$ with the norm $\|f\|$ $=\sup \left\{\left|f(z) e^{1 / m|z|}\right| ; z \in U_{m} \cap \boldsymbol{C}\right\}$.

DEFINITION 3.4. We set $\mathscr{P}_{*}=\mathscr{Q}(\boldsymbol{D})$.

REMARK 3.1. $\mathscr{P}_{*}$ becomes a DFS-space ([13])。 Furthermore, it is a subspace of $L^{2}$ and stable under the Fourier transformations.

DEFINITION 3.5. For any compact set $K$ in $R$, we denote by $\mathscr{B}_{K}(R)$ the set of all hyperfunctions on $\boldsymbol{R}$ with the supports in $K$.

As an example of such hyperfunctions, we give Dirac's delta-function $\delta$ as follows :

$$
\delta=\left[-\frac{1}{2 \pi i z}, D \times \sqrt{-1} R\right],
$$

that is, $\delta$ is a Fourier hyperfunction on $D$ with the defining function $\left(-\frac{1}{2 \pi i z}, D \times \sqrt{-1} R-\{0\}\right)$ and its support in $\{0\}$.

Now, we shall give the definition of local operators on $\mathscr{R}$. Let $S$ be any entire function of infra-exponential type, i.e., satisfying the condition that for any $\varepsilon>0$ there exists some $c_{\varepsilon}>0$ and the estimate

$$
|S(z)| \leqslant c_{s} e^{\varepsilon z \mid} \quad \text { for any } z \in C
$$

holds. Let $S(z)$ be expanded into a Taylor series

$$
S(z)=\sum_{n=0}^{\infty} a_{n}(i z)^{n} .
$$

We then note that (3.5) is equivalent to

$$
\lim _{n \rightarrow \infty} \sqrt[n]{n !\left|a_{n}\right|}=0
$$

Given such a function $S$. By (3.6) and Cauchy's integral formula, since for any open set $V$ in $\boldsymbol{D} \times \sqrt{-1} \boldsymbol{R}$ there exists an open set $V_{1} \subset V$ such that

$$
S\left(\frac{1}{i} \frac{d}{d z}\right) \varphi(z) \equiv \sum_{n=0}^{\infty} a_{n} \varphi^{(n)}(z) \in \tilde{\mathscr{O}}\left(V_{1}\right), \quad \text { for any } \varphi \in \tilde{\mathscr{O}}(V),
$$


we can define a linear operator $S\left(\frac{1}{i} \frac{d}{d x}\right)$ from $\mathscr{R}$ into $\mathscr{R}$ as follows: for any open set $D$ in $D$ and any $\mu=[\varphi, V] \in \mathscr{R}(D)$,

$$
S\left(\frac{1}{i} \frac{d}{d x}\right) \mu=\left[S\left(\frac{1}{i} \frac{d}{d z}\right) \varphi(z), V_{1}\right]
$$

Then, it is clear that the operator $S\left(\frac{1}{i} \frac{d}{d x}\right)$ has the local property in the sence that for any $\mu \in \mathscr{R}(D)$ and any open set $D_{1}$ in $D$ if $\mu=0$ in $D_{1}$, then $S\left(\frac{1}{i} \frac{d}{d x}\right) \mu=0$ in $D_{1}$.

DEFINITION 3.6. We call this operator $S\left(\frac{1}{i} \frac{d}{d x}\right)$ a local operator in $\mathscr{R}$.

Moreover we define $\varphi \in \mathcal{O}(\boldsymbol{C}-\{0\})$ by

$$
\varphi(z)=S\left(\frac{1}{i} \frac{d}{d z}\right)\left(-\frac{1}{2 \pi i z}\right), z \in C-\{0\} .
$$

We then see that it satisfies

$$
\varphi \in \mathcal{O}(\boldsymbol{C}-\{0\}) \text { and } \lim _{|z| \rightarrow \infty} \varphi(z)=0 .
$$

Therefore, defining $\mu \in \mathscr{R}(D)$ with the defining function $(\varphi, R \times \sqrt{-1} R)$, we obtain, by (3.4) and (3.7),

$$
\mu=S\left(\frac{1}{i} \frac{d}{d x}\right) \delta \quad \text { in } R
$$

Conversely, let $\mu$ be any element of $\mathscr{B}_{\{0\}}(\boldsymbol{R})$ and $\varphi(\in \mathcal{O}(\boldsymbol{C}-\{0\}))$ be its standard defining function ([19]). Then, $\varphi$ satisfies (3.9) and is characterized by it. Hence, by expanding $\varphi$ into a Laurent series, we can prove that there uniquely exists an entire function $S_{\mu}$ of infra-exponential type such that (3.8) and (3.10) hold for $S=S_{\mu}$. Thus, we have

THEOREM 3.1 (The structure theorem of $\mathscr{B}_{[0\}}(\boldsymbol{R})$ ) ([22]). For any $\mu \in$ $\mathscr{B}_{(0\}}(\boldsymbol{R})$, there exists a local operator $S\left(\frac{1}{i} \frac{d}{d x}\right)$ in $\mathscr{R}$ such that $\mu=$ $S\left(\frac{1}{i} \frac{d}{d x}\right) \delta$ in $R$.

Next we shall define a Fourier transformation on $\mathscr{R}(D)$. For this, we define a linear operator $J$ from $\mathscr{R}(D)$ into $\mathscr{P}_{*}^{\prime}$ as follows: for any $\mu=[\varphi, V]$ $\in \mathscr{R}(\boldsymbol{D})$ and any $f \in \mathscr{P}_{*}$ 


$$
\langle J \mu, f\rangle=\int_{R} \varphi(x+i \varepsilon) f(x+i \varepsilon) d x-\int_{R} \varphi(x-i \varepsilon) f(x-i \varepsilon) d x,
$$

where $\varepsilon$ is sufficiciently small positive number. It can be shown that (3.11) is well-defined, independent of $\varepsilon$ and it gives an element of $\mathscr{P}_{*}^{\prime}$. Then, it can be proved that $J$ is bijective ([12]). Since $\hat{f} \in \mathscr{P}_{*}$ for any $f \in \mathscr{P}_{*}$ by Remark 3.1, we can define an operator $\mathscr{F}$ from $\mathscr{P}_{*}^{\prime}$ onto $\mathscr{P}_{*}^{\prime}$ by

$$
\left\langle\mathscr{F} T, f^{\prime}\right\rangle=\langle T, \hat{f}\rangle, \text { for any } \mathbb{T}^{\top} \in \mathscr{P}_{*}^{\prime} \text { and any } f \in \mathscr{P}_{*} \text {. }
$$

DEFINITION 3.7. The operator $\mathscr{F} \mathscr{R}=J^{-1} \mathscr{J}$ from $\mathscr{R}(D)$ onto $\mathscr{R}(\mathbb{D})$ IS called a Fourier transformation on $\mathscr{R}(\boldsymbol{D})$.

After above preparations, we shall prove some lemmas which are necessary in the coming sections.

At first, we shall give concrete imbeddings of $\widetilde{L^{j}}$ into $\mathscr{R}(D)$, where $\widetilde{L^{j}}$ means the inverse Fourier image of $L^{j}$-spaces $(j=1,2)$. For any $u=\tilde{f} \in \widetilde{L^{j}}$ $(j=1,2)$, we define $\varphi_{u}(z)(z \in C-R)$ by

$$
\varphi_{u}(z)=\left\{\begin{aligned}
\frac{1}{2 \pi} \int_{0}^{\infty} e^{i z t} f(t) d t, & z \in C^{+}, \\
-\frac{1}{2 \pi} \int_{-\infty}^{0} e^{i z t} f(t) d t, & z \in C^{-}
\end{aligned}\right.
$$

Then, noting that $\left|(i t)^{n} e^{i z t} f(t)\right| \leq|t|^{n} e^{-\delta|t|}|f(t)|$ for any $\delta>0$, any $n \in N_{*}$ and any $z \in \boldsymbol{C}-\boldsymbol{R},|\operatorname{Im} z| \geq \delta$, we see from Lebesgue's dominated convergence theorem that

LEMMA 3.1. For any $u=\tilde{f} \in \widetilde{L^{1}}$,

(i) $\varphi_{u} \in \mathcal{O}(\boldsymbol{C}-\boldsymbol{R})$ and $\left|\varphi_{u}(z)\right| \leq \frac{1}{2 \pi} \int_{\boldsymbol{R}}|f(t)| d t, z \in \boldsymbol{C}-\boldsymbol{R}$,

(ii) for any $x \in R, \lim _{\varepsilon \downarrow 0} \varphi_{u}(x \pm i \varepsilon) \equiv \varphi_{u}(x \pm i 0)$ existand $\varphi_{u}(x+i 0)-\varphi_{u}(x-i 0)$ $=u(x)$.

Similarly, we can prove

LEMMA 3.2. For any $u=\tilde{f} \in \widetilde{L^{2}}$,

(i) $\varphi_{u}(z)=\frac{1}{2 \pi i} \int_{\boldsymbol{R}} \frac{u(t)}{t-z} d t, z \in \boldsymbol{C}-\boldsymbol{R}$,

(ii) $\varphi_{u} \in \mathcal{O}(\boldsymbol{C}-\boldsymbol{R})$ and is bounded in $\{z \in C ;|\operatorname{Im} z| \geq \delta\}$ for any $\delta>0$,

(iii) $\varphi_{u}^{(n)}(\cdot+i y) \in L^{2}$, for any $n \in N_{*}$ and any $y \in \mathbb{R}-\{0\}$;

(iv) l.i.m. $\varphi_{\varepsilon \downarrow 0}(\cdot \pm i \varepsilon) \equiv \varphi_{u}(\cdot \pm i 0)$ exist and $\varphi_{u}(\cdot+i 0)-\varphi_{u}(\cdot-i 0)=u(\cdot)$,

(v) for any $n \in N_{*}$ and any $z_{0} \in C-\boldsymbol{R}$

$$
\lim _{\boldsymbol{C}-\boldsymbol{R} \ni z \rightarrow z_{0}}\left\|\frac{\varphi_{u}^{(n)}(z-\cdot)-\varphi_{u}^{(n)}\left(z_{0}-\cdot\right)}{z-z_{0}}-\varphi_{u}^{(n+1)}\left(z_{0}-\cdot\right)\right\|_{L^{2}}=0 .
$$


As an application of Lemmas 3.1 and 3.2, we shall give a complexified representation of (2.21). Since $K \subset \widetilde{L^{1}}$ by (2.21), we can define $\varphi_{u}$ by (3.13) for any $u \in \mathbb{K}$. We then claim that for any $u \in \mathbb{K}$ and any $z \in C-$ 踏

$$
\varphi_{u}(z)=\frac{1}{\sqrt{2 \pi}} \int_{R} \varphi_{E}(z-s) K u(s) d s .
$$

In fact, this integral is well-defined by (2.17) and Lernma 3.2 (iii). Furthermore, it follows from (2.21) that $u=\frac{1}{\sqrt{2 \pi}}(\hat{E} \widehat{K u})^{\sim}$ for any $u \in \mathbb{K}$. Therefore, noting (3.13), we obtain (3.14).

By Lemmas 3.1 (i) and 3.2 (ii), we can define linear operators $l_{j}$ from $\widetilde{L^{j}}$ into $\mathscr{R}(\boldsymbol{D})$ by

$$
l_{j} u=\left[\varphi_{u}, D \times \sqrt{-1} R\right], \quad \text { for any } u \in \widetilde{L^{j}}(j=1,2) .
$$

Then we shall show

LEMMA 3.3. For any $j \in\{1,2\}, l_{j}$ is local in the sense that, for any open set $D$ in $D$ and any $u \in \widetilde{L^{j}}$, if $u=0$ in $D \cap R$, then $l_{j} u=0$ in $D$.

Proor. Since $\mathscr{R}$ is a sheaf, it is enough to consider the case where $D$ is an interval with left end point $a$ and right end point $b$. When both $a$ and $b$ are finite, Lemma 3.3 follows from Lemmas 3.1 (ii) and 3.2 (iv). When $a$ is $-\infty$ and $b$ is $\infty$, Lemma 3.3 is trivial since $\varphi_{u}=0$ by (3.13). Therefore, we shall consider the case where $a$ is finite and $b$ is $\infty$. The rest is proved similarly. Let $u$ be any element of $\widetilde{L^{j}}$ such that $u=0$ in $(a, \infty)$. By Lemma 3.1 (i) (ii) or Lemma 3.2 (i) (iv), we see that $\varphi_{u} \in$ $\mathcal{O}(\operatorname{Re} z>a)$ and it is bounded in $\{z \in C ; \operatorname{Re} z \geq a+\varepsilon\}$ for any $\varepsilon>0$. This implies that $\varphi_{u} \in \tilde{\mathscr{O}}((a, \infty] \times \sqrt{-1} R)$ and so $l_{j} u=0$ in $(a, \infty]$.

(Q.E.D.)

For later use, we shall prove

LEMMA 3.4. Let $S$ be any entire function of infra-exponential type. For each $j \in\{1,2\}$ and any $u=\tilde{f} \in \widetilde{L^{j}}$,

(i) $S\left(\frac{1}{i} \frac{d}{d x}\right) l_{j} u=\left[S\left(\frac{1}{i} \frac{d}{d z}\right) \varphi_{u}(z), D \times \sqrt{-1} R\right]$,

(ii) $S\left(\frac{1}{i} \frac{d}{d z}\right) \varphi_{u}(z)=\left\{\begin{array}{cc}\frac{1}{2 \pi} \int_{0}^{\infty} e^{i z t} S(t) f(t) d t, & z \in \mathbb{C}^{+}, \\ -\frac{1}{2 \pi} \int_{-\infty}^{0} e^{i z t} S(t) f(t) d t, & z \in C^{-},\end{array}\right.$

(iii) $S\left(\frac{1}{i} \frac{d}{d z}\right) \varphi_{u}(z)$ is bounded in $\{z \in C ;|\operatorname{Im} z| \geq \varepsilon\}$ for any $\varepsilon>0$.

Proof. (i) follows from (3.7) and (3.15). Since $S\left(\frac{1}{i} \frac{d}{d z}\right)$ is a differential operator with constant coefficients satisfying (3.6), (3.13) yields (ii). Noting that $S$ satisfies (3.5), (iii) follows from (ii).

(Q.E.D.) 
Finally, in connection with the Fonrier transformation F on 2 and the Fourier transformation of on $9(0)$, we shall give

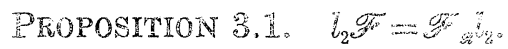

Proor. Let $f$ and $\varphi$ be any element of $L^{2}$ whe $Q_{*}$, respectively. $B g$ (3.11), (8.15) and Lemma s.2 (0), ror suffictembly smat $s>0$,

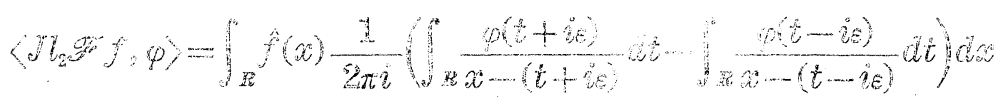

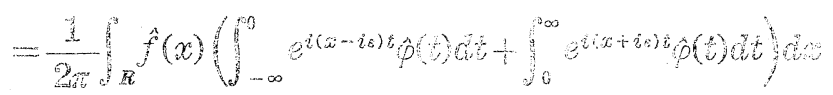

Letting $\varepsilon$ tend to zero, we obtain

$$
\left\langle H_{2} \mathscr{J}, f, p\right\rangle=\int_{z i} f(x) \psi(w) d x
$$

On the other hand, by (3.11), (3.12), (8.15) and Lemma s.8 (0),

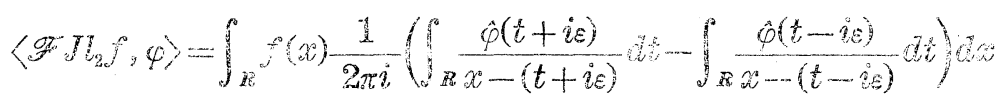

$$
\begin{aligned}
& =\int_{R} f(x)\left(\int_{-\infty}^{0} e^{i\left(x-i_{0}\right) t} \rho(-t) d t+\int_{0}^{\infty} e^{i\left(x+i_{0}\right) t} \varphi(-b) d t\right) d x
\end{aligned}
$$

for suficiently small $\varepsilon>0$, Leting $\varepsilon$ tend to zero, we have

$$
\left\langle\mathscr{F} J l_{2} \hat{f}^{\circ}, \varphi\right\rangle=\int_{R} f(x) \hat{\varphi}(x) d x=\int_{I R} \hat{f}(x) \varphi(x) d x 。
$$

Therefore, by (3.1.6) and (3.17), we set that $J_{2} \mathscr{F}=\mathscr{F} J l_{2}$ and so $l_{2} \mathscr{F}=\mathscr{F} l_{2}$ by Definition 3.7.

As an application of Proposition 3.1, we shall show that $\mathbb{E}$ in (2.15) can be recaptured from the point of view of Tourier hyperfunctions. Since $h(z)\left(z \in C^{+}\right)$in (2.11) satisfies (2.12), it follows from Hille-Tamarkin's theorem ([8]) that for any $\varepsilon>0$ the estimate $\sup \{|h(z)| ; \operatorname{Im} z \geq \varepsilon\}<\infty$ holds. Therefore, we can define a Fourier hyperfunction $\mu_{h}(\in \mathscr{R}(D))$ whose defining function is $h(z)$ in $C^{+}$and zero in $C^{-}$:

$$
\mu_{h}=[\varepsilon(z) h(z), D \times \sqrt{-1} R],
$$

where $\varepsilon(z)=1$ in $C^{+}$and 0 in $C^{-}$. Then we claim that

$$
l_{2} E=\mathscr{F}_{a} \mu_{h} \text {. }
$$

In fact, since $h(z)\left(z \in C^{+}\right)$satisfies (2.12), it follows from Paley-Wiener's theorem ([9], [17]) that 


$$
\frac{1}{2 \pi i} \int_{R} \frac{h(\lambda)}{\lambda-z} d \lambda=\left\{\begin{array}{cc}
h(z), & z \in C^{+}, \\
0, & z \in C^{-},
\end{array}\right.
$$

when $h(\lambda)(\lambda \in R)$ is an $L^{2}$-function in (2.13). Therefore, by Lemma 3.2 (i) and (3.18), we see that $l_{2} h=\mu_{h}$. Thus, it follows from (2.15), (3.13) and Proposition 3.1 that $l_{2} E=l_{2} \mathscr{F} h=\mathscr{F}_{\mathscr{R}} l_{2} h=\mathscr{F}_{\mathscr{R}} \mu_{h}$.

\section{§4. Symbol of $K$}

In this section we shall give a necessary and sufficient condition that the operator $K$ in Proposition 2.5 has the local property.

Given any complex valued measurable function $S$ in $\boldsymbol{R}$, we define linear operators $T_{s}^{j}$ from $\widetilde{L^{1}}$ into $\widetilde{L^{j}}(j=1,2)$.

Definition 4.1. For each $j \in\{1,2\}$, we set

$$
D\left(T_{s}^{j}\right)=\left\{u \in \widetilde{L}^{1} ; S \hat{u} \in L^{j}\right\} \quad \text { and } \quad T_{s}^{j} u=\frac{1}{\sqrt{2 \pi}} \widetilde{S \hat{u}}
$$

where $\hat{u}$ is a unique element of $L^{1}$ such that $\widetilde{\hat{u}}=u$.

We call a function $S$ a symbol of the operators $T_{s}^{j}(j=1,2)$. In particular, when $S$ is equal to $\check{h}^{-1}, T_{s}^{j}$ are denoted by $K_{j}(j=1,2)$. By Proposition 2.5 , it is easy to see that the operator $K_{2}$ is a complexification of $K$, that is,

$$
D\left(K_{2}\right)=K+\sqrt{-1} K \text { and } u=\frac{1}{\sqrt{2 \pi}} E * K_{2} u
$$

As usual, we define for each $a \in \boldsymbol{R}$ a translation operator $\tau_{a}$ by $\left(\tau_{a} f\right)(\cdot)$ $=f(\cdot+a)$. Then it is clear that, for any $a \in R$ and each $j \in\{1,2\}, T_{s}^{j}$ is $\tau_{a^{-}}$ commutative, i.e.,

$$
\tau_{a}\left(D\left(T_{s}^{j}\right)\right) \subset D\left(T_{s}^{j}\right) \quad \text { and } \quad \tau_{a} T_{s}^{j}=T_{s}^{j} \tau_{a} .
$$

Considering that the spaces $\widetilde{L^{j}}$ are Banach spaces with the norms $\|u\|_{\tilde{L}^{j}}$ $=\|\hat{u}\|_{L^{\prime}}(j=1,2)$, we can easily prove

LEMMA 4.1. $\quad T_{\mathrm{s}}^{j}$ are closed linear operators $(j=1,2)$.

Similarly as in Definition 2.5, we give

Definition 4.2. For each $j \in\{1,2\}, T_{s}^{j}$ is said to be local if $u \in D\left(T_{s}^{j}\right)$ and $u=0$ in $(a, b)$, then $T_{s}^{j} u=0$ in $(a, b)(-\infty<a<b<\infty)$.

REMARK 4.1. By (2.21) and (4.2), the local property of $K$ is equivalent to the one of $K_{2}$.

Next we define for each $a \in \boldsymbol{R} u_{a} \in \widetilde{L^{1}}$ by

$$
u_{a}=\left(e^{-i a \cdot h} \frac{1}{(1+i \cdot)^{2}}\right)^{\sim}
$$


Then we shall show

LEMMA 4.2. For each $a \in \mathbb{R}$

(i) $u_{a} \neq 0, \quad u_{a} \in L^{2} \cap D\left(K_{j}\right)(j=1,2)$,

(ii) $u_{a}=0$ in $(-\infty, a)$.

PRoof. (i) follows from (2.14), (4.1) and (4.4). Furthermore, by (4.4)

$$
u_{a}(t)=(2 \pi)^{-1} \int_{R} E(t-a-s)\left(\frac{1}{\left(1+i_{0}\right)^{2}}\right)^{\sim}(s) d s, \quad \text { for any } a \in \mathbb{R} \text {. }
$$

Since $\frac{1}{(1-i \cdot)^{2}}$ belongs to $H^{2+},\left(\frac{1}{(1+i \cdot)^{2}}\right)^{\sim}$ vanishes on $(-\infty, 0)$. Therefore, noting (2.16), we have (ii).

Using Lemma 4.2, we shall prove

Propositron 4.1. For each $j \in\{1,2\}, K_{j}$ is a closed linear operator with dense domain.

Proof. By Lemma 4.1, it suffices to show that each $K_{j}(j=1,2)$ has a dense domain. For this, by Lemma 4.2, we have only to see that $\left\{u_{a} ; a \in \mathbb{R}\right\}$ is dense in $\widetilde{L^{1}}$. Consider any $\Phi \in\left(\widetilde{L^{1}}\right)^{\prime}$ such that $\Phi\left(u_{a}\right)=0$ for any $a \in \boldsymbol{R}$. We then define $\hat{\Phi} \in\left(L^{1}\right)^{\prime}$ by $\hat{\Phi} f=\Phi(\tilde{f})$. Since $\left(L^{1}\right)^{\prime}=L^{\infty}$, there exists a bounded measurable function $\varphi$ such that $\hat{\Phi} f=\int_{R} f(\lambda) \varphi(\lambda) d \lambda$ for any $f \in L^{1}$. Therefore, by (4.4),

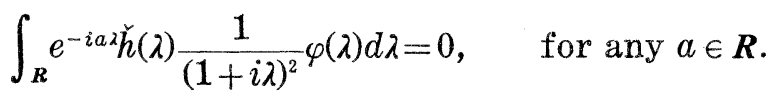

Noting (2.14), this implies that $\varphi=0$ and hence $\Phi=0$. Thus, we have proved Proposition 4.1.

Now, by virtue of Lemma 4.2, we can apply Theorem 1 in L. de Branges [3] to our operator $K_{1}$ and get

THEOREM 4.1 ([3]). The operator $K_{1}$ is local if and only if its symbol $\check{h}^{-1}$ is an entire function of infra-exponential type.

Noting Lemma 4.2 and using the same method as in Theorem 4.1, we can show an $L^{2}$-version of Theorem 4.1 , that is,

THEOREM 4.2. The operator $K_{2}$ is local if and only if its symbol $\check{h}^{-1}$ is an entire function of infra-exponential type.

REMARK 4.2. For any $u \in \widetilde{L^{1}}$ and any $\varphi \in C_{0}^{\infty}(\boldsymbol{R})$, we define $v=\varphi * u$. If $u \in D\left(K_{2}\right)$, then $v \in D\left(K_{1}\right)$ and $K_{1} v=\varphi * K_{2} u$. Therefore, it is easy to see directly that if $K_{1}$ is local so is $K_{2}$.

By using the theory of M. Sato's hyperfunctions, we shall give another proof of Theorems 4.1 and 4.2. For this, we define a subspace $Z_{\Delta}$ of $Z_{\Delta}$ by 
(4.5) $\quad Z_{4}=$ the set of all elements of $Z_{4}$ which are the restrictions to $R$ of entire functions of infra-exponential type.

The following Lemma 4.3 is proved in [14].

LEMMA 4.3 ([14]). $\partial Z_{\Delta}(0) \subset Z_{d}$, for any Hardy weight $\Delta$.

REMARK 4.3. It is also proved in [14] that for any weight $\Delta$ (Hardy or not)

$$
Z_{\Delta} \subset \partial Z_{4}(0) .
$$

The proof of (4.6) in [14] is not easy. In case $\Delta$ is a Hardy weight, H. Dym and H. P. McKean, Jr. [6] gave another proof of (4.6) using the theory of de Branges spaces ([4]). In the last part of this section, we shall give a simple froof (4.6) using the theory of M. Sato's hyperfunctions.

The proof of necessity of Theorems 4.1 and 4.2: Consider $u_{0}$ for $a=0$ in (4.4). We then claim that for any $\varepsilon>0$

$$
\check{h}^{-1} \hat{u}_{0} \in \text { the closed linear hull of }\left\{e^{i t} \cdot \hat{u}_{0} ;|t|<\varepsilon\right\} \text { in } L^{2} \text {. }
$$

We note that both $\check{h}^{-1} \hat{u}_{0}$ and $\hat{u}_{0}$ belong to $L^{2}$ by Lemma 4.2 (ii). For the proof of (4.7), take any $g \in L^{2}$ such that $\left(e^{i t} \cdot \hat{u}_{0}, g\right)_{L^{2}}=0$ for any $t \in(-\varepsilon, \varepsilon)$. We define $u \in \widetilde{L^{1}}$ by $u=\widetilde{u_{0} \widetilde{g}}$. Then, $u=0$ in $(-\varepsilon, \varepsilon)$. Therefore, since $u \in D\left(K_{j}\right)(j=1,2)$ by $(4.4)$, the local property of $K_{1}$ (resp. $K_{2}$ ) implies that $K_{1} u=0$ in $(-\varepsilon, \varepsilon)$ (resp. $K_{2} u=0$ in $(-\varepsilon, \varepsilon)$ ). But, noting that $\check{h}^{-1} \hat{u} \in L^{1}$ by (4.4), we see that $\left(\breve{h}^{-1} \hat{u}_{0}, g\right)_{L^{2}}=0$ and so (4.7) holds. Now, (4.7) yields that there exists a sequence $\left(P_{n}\right)_{n=1}^{\infty}$ for which each $P_{n}$ is a finite linear combination of $e^{i t .},|t|<\frac{1}{n}$ and $\operatorname{lii.m}_{n \rightarrow \infty} P_{n} \hat{u}_{0}=\check{h}^{-1} \hat{u}_{0}$. Since $\left|\hat{u}_{0}\right|^{2}=\frac{\Delta}{\left(1+\lambda^{2}\right)^{2}}$ by (2.13) and (4.4), this implies that $\check{h}^{-1} \in \partial Z_{\Delta /\left(1+k^{2}\right) 2}(0)$. Hence, applying Lemma 4.3 to this Hardy weight $\frac{\Delta}{\left(1+\lambda^{2}\right)^{2}}$, we see that $\check{h}^{-1}$ is an entire function of infraexponential type.

The proof of sufficiency of Theorems 4.1 and 4.2: For that purpose we shall show a more general

THEOREM 4.3. For any entire function $S$ of infra-exponential type and each $j \in\{1,2\}, T_{s}^{j}$ is local.

Proof. Take any $u \in D\left(T_{s}^{j}\right)$ such that $u=0$ in $(a, b)$. It follows from Lemma 3.3 that $l_{j} u=0$ in $(a, b)$ and so $S\left(\frac{1}{i} \frac{d}{d x}\right) l_{j} u=0$ in $(a, b)$. Since $u \in D\left(T_{s}^{j}\right)$ yields $S \hat{u} \in L_{j}$, we see from Lemmas $3.1,3.2$ and 3.4 (i) (ii) that $\widetilde{S \hat{u}}=0$ in $(a, b)$. This completes the proof of Theorem 4.3.

(Q.E.D.) 
Finally we shall prove (4.6) as an application of Theorem 4.3.

Proof of (4.6): Fix any $S \in Z_{\dot{4}}$. By Theorem 4.3, we know that $T_{S}^{1}$ is local. Therefore, noting $S \Delta^{1 / 2} \in L^{2}$, exactly in the same way as in the proof of (4.7), we see that for any $\varepsilon>0 S \Delta^{1 / 2}$ belongs to the closed linear hull of $\left\{e^{i t \cdot} \Delta^{1 / 2} ;|t|<\varepsilon\right\}$ in $L^{2}$. This implies that $S \in \partial Z_{\Delta}(0)$.

(Q.E.D.)

\section{§ 5. Markovian property of $X$ and local property of $K$}

As stated in $\S 1$, in connection with a special Bernstein's problem about trigonometrical approximations in the space $Z_{\Delta}, \mathrm{N}$. Levinson and H.P. McKean, Jr. [14] have proved

THEOREM 5.1 ([14]). The conditions (i) and (ii) are equivalent:

(i) The process $\boldsymbol{X}$ has the Markovian property;

(ii) The outer function $h$ of the spectral density $\Delta$ is an inverse of an entire function of infra-exponential type.

For the purpose of revealing the mechanism of Theorem 5.1, we have introduced the unitary operator $K$ from $K$ onto $L^{2}(\boldsymbol{R})$. Combining Theorem 2.1 with Theorem 4.2, it is clarified that (i) implies (ii) in Theorem 5.1. That is, we find that the Markovian property of $\boldsymbol{X}$ implies the local property of $K$ and then the later yields that the symbol $\breve{h}^{-1}$ of $K$ is an entire function of infra-exponential type. The proof in [14] that (i) implies (ii) in Theorem 5.1 is as follows: calculating for any $t>0$ the projection of $e^{i t}$ upon $Z_{\Delta}^{-}(0)$, it follows from Proposition 2.8 and Lemma 4.3 that for any $t>0$

$$
f_{t} \equiv e^{-i t \cdot h^{-1}} \int_{t}^{\infty} e^{i \cdot s} E(s) d s \in Z_{\dot{4}}
$$

Next, using the compactness lemma in $Z_{\dot{4}}$, it can be shown that for any $t>0$ there exist some $f \in Z_{\Delta}$ and some positive sequence $\left(\delta_{n}\right)_{n=1}^{\infty}$ tending to zero for which $\lim _{n \rightarrow \infty} . f_{t+\delta_{n}}=f$ in $Z_{\dot{\Delta}}$. Therefore, choosing an appropriate $t>0$, (ii) can be proved.

In this section, using the theory of M. Sato's hyperfunctions, we shall show that (ii) implies (i) in Theorem 5.1. For that purpose, by Proposition 2.2, we have only to prove the following conditions (5.1) and (5.2):

$$
\begin{gathered}
\boldsymbol{K}^{-}(0) \cap \boldsymbol{K}^{+}(0)=\boldsymbol{K}^{+/-}(0) ; \\
\partial \boldsymbol{K}(0)=\boldsymbol{K}^{-}(0) \cap \boldsymbol{K}^{+}(0) .
\end{gathered}
$$

By Corollary 2.2, (5.1) (resp. (5.2)) is equivalent to the next (5.3) (resp. (5.4)) :

$$
Z_{\Delta}^{-}(0) \cap Z_{\Delta}^{+}(0)=Z_{\Delta}^{+/-}(0)
$$




$$
\partial Z_{\Delta}(0)=Z_{\Delta}^{-}(0) \cap Z_{\Delta}^{+}(0)
$$

N. Levinson and H. P. McKean, Jr. have proved (5.3) showing that (5.3) is valid if and only if $\check{h}^{-1} h$ can be extended to holomorphic function on $\boldsymbol{C}^{+}$whose absolute value is less than one. Furthermore they have proved (5.4) showing that if $\Delta$ is a Hardy weight and its inverse is locally integrable, then (5.4) holds. The latter follows from the steps that each element of $\boldsymbol{Z}_{\Delta}^{-}(0) \cap \boldsymbol{Z}_{\Delta}^{+}(0)$ can be extended to an entire function, and then it is found to be of infra-exponential type using Phragmen-Lindelöf's theorem and so it belongs to $\partial Z_{4}(0)$ by (4.6).

On the other hand, we shall give a direct proof of (5.1) and (5.2) in the sequel. Its outline is as follows: (5.1) can be easily shown by Theorem 4.2. For the proof of (5.2), we first construct for each $u \in \boldsymbol{K}^{-}(0) \cap \boldsymbol{K}^{+}(0)$ an entire function $S_{u}$ of infra-exponential type with the aid of the structure theorem of $\mathscr{B}_{\{0\}}(R)$ (Theorem 3.1). We then prove that $S_{u}$ is an element of $Z_{\Delta}$ corresponding to $u$ under the unitary operator $U_{2}$ (Proposition 2.7) by using the Fourier transformation in $\mathscr{R}(D)$. This implies that $u \in \partial K(0)$ by Corollary 2.2 and (4.6). As an application of our proof, we shall get a concrete form of each element of $\partial Z_{4}(0)$. By means of this form, we shall obtain an explicit formula of the predictor using the past.

Now we suppose in the sequel that there exists an entire function $P$ of infra-exponential type for which

$$
\check{h}^{-1}=P \text {. }
$$

We then define an entire function $Q$ of infra-exponential type by

$$
Q=P \check{P} \text {. }
$$

The proof of (5.1): We have only to prove that $K^{+}(0)^{\perp} \subset K^{-}(0)$, for it is equivalent to (5.1). Fix any $u \in K^{+}(0)^{\perp}$. By Lemma 2.2 (i), $u=0$ in $(0, \infty)$. Since the operator $K$ is local by Remark 4.1 and Theorem 4.2, we see that $K u=0$ in $(0, \infty)$. By Lemma 2.12 (ii), this implies that $u \in K^{-}(0)$. Thus, we have proved (5.1).

The proof of (5.2): By Lemma 2.6 (ii), it suffices to show that $K^{-}(0) \cap$ $K^{+}(0) \subset \partial K(0)$. It follows from (2.15), (2.21) and (2.22) that for any $u \in K$

$$
u=\sqrt{2 \pi}(\check{h}, \widehat{K u})^{\sim}=\sqrt{2 \pi}(h, \widehat{\tilde{K} u})^{\sim} .
$$

Using Lemmas 3.1, 3.2 and 3.4, we see from (5.5) and (5.7) that for any $u \in \boldsymbol{K}$ 


$$
P\left(\frac{1}{i} \frac{d}{d x}\right) l_{1} u=\sqrt{2 \pi} l_{2} K u \text { and } \check{P}\left(\frac{1}{i} \frac{d}{d x}\right) l_{1} u=\sqrt{2 \pi} l_{2} \check{K} u
$$

In particular, consider any $u \in K^{-}(0) \cap K^{+}(0)$ and fix it. By Lemmas 2.12 (ii) and 2.13 (iii),

$$
K u=0 \text { in }(0, \infty) \text { and } \check{K} u=0 \text { in }(-\infty, 0) \text {. }
$$

Furthermore, using Lemma 3.3, we have

$$
l_{2} K u=0 \text { in }(0, \infty] \text { and } l_{2} \check{K} u=0 \text { in }[-\infty, 0) \text {. }
$$

Therefore, by operating two local operators $P\left(\frac{1}{i} \frac{d}{d x}\right)$ and $P\left(\frac{1}{i} \frac{d}{d x}\right)$ to the first term and the second term in (5.8), respectively and using Lemma 3.4, we see from (5.7) and (5.10) that

$$
Q\left(\frac{1}{i} \frac{d}{d x}\right) l_{1} u=0 \quad \text { in }[-\infty, 0) \cup(0, \infty] .
$$

Especially, since (5.11) implies that $Q\left(\frac{1}{i} \frac{d}{d x}\right) l_{1} u \in \mathscr{B}_{\{0\}}(\boldsymbol{R})$, it follows from Theorem 3.1 that there exists an entire function $S_{u}$ of infra-exponential type for which

$$
Q\left(\frac{1}{i} \frac{d}{d x}\right) l_{1} u=2 \pi \check{S}_{u}\left(\frac{1}{i} \frac{d}{d x}\right) \delta \quad \text { in } \quad \boldsymbol{R} \text {. }
$$

Since by Lemma 3.4 (i) $Q\left(\frac{1}{i} \frac{d}{d z}\right) \varphi_{u}(z)$ is a defining function of the hyperfunction $Q\left(\frac{1}{i} \frac{d}{d x}\right) l_{1} u$, we see that the entire function $S_{u}$ can be expressed in the form

$$
S_{u}(\cdot)=\sum_{n=0}^{\infty} a_{n}(i \cdot)^{n} \quad \text { and } \quad a_{n}=-\frac{1}{2 \pi n !} \oint z^{n} Q\left(\frac{1}{i} \frac{d}{d z}\right) \varphi_{u}(z) d z
$$

where the integral in (5.13) is taken on a sufficiently small sphere with the centre 0 (the origin of $C$ ) and its value is independent of such a sphere.

Next we claim that (5.12) holds in $\boldsymbol{D}$, that is, it is valid as Fourier hyperfunctions. Since $Q\left(\frac{1}{i} \frac{d}{d z}\right) \varphi_{u}(z)$ is a defining function of the Fourier hyperfunction $Q\left(\frac{1}{i} \frac{d}{d x}\right) l_{1} u$, by (5.11), we have only to prove that the holo- 
morphic part in Laurent's expansion of $Q\left(\frac{1}{i} \frac{d}{d z}\right) \varphi_{u}(z)$ around the origin belongs to $\tilde{\mathscr{O}}(\boldsymbol{D})$. Using Lemma 3.4, we see from (5.5), (5.6), (5.7) and (5.9) that

$$
Q\left(\frac{1}{i} \frac{d}{d z}\right) \varphi_{u}(z)= \begin{cases}\frac{1}{\sqrt{2 \pi}} \int_{-\infty}^{0} K u(s)\left(\int_{0}^{\infty} e^{i(z-s) t} P(-t) d t\right) d s, & z \in C^{+}, \\ -\frac{1}{\sqrt{2 \pi}} \int_{0}^{\infty} \check{K} u(s)\left(\int_{-\infty}^{0} e^{i(z-s) t} P(t) d t\right) d s, & z \in C^{-} .\end{cases}
$$

Since $P$ is an entire function of infra-exponential type, it has the form

$$
P(\cdot)=\sum_{n=0}^{\infty} c_{n}(-i \cdot)^{n} \quad \text { and } \quad \lim _{n \rightarrow \infty} \sqrt[n]{\left|c_{n}\right| n !}=0 .
$$

Substituting (5.15) into (5.14), we find that

$$
Q\left(\frac{1}{i} \frac{d}{d z}\right) \varphi_{u}(z)=\left\{\begin{array}{l}
\frac{1}{\sqrt{2 \pi} i} \int_{-\infty}^{0} K u(s)\left(\sum_{n=0}^{\infty} c_{n} n ! \frac{1}{(s-z)^{n+1}}\right) d s, \quad z \in C^{+}, \\
\frac{1}{\sqrt{2 \pi} i} \int_{0}^{\infty} \check{K} u(s)\left(\sum_{n=0}^{\infty} c_{n}(-1)^{n} n ! \frac{1}{(s-z)^{n+1}}\right) d s, \quad z \in C^{-} .
\end{array}\right.
$$

Therefore, by (5.15), we see that $Q\left(\frac{1}{i} \frac{d}{d z}\right) \varphi_{u}(z)$ belongs to $\mathcal{O}(C-\{0\})$ and it is bounded in $\{z \in C ;|z| \geq \varepsilon\}$ for any $\varepsilon>0$. This implies that the holomorphic part in Laurent's expansion of $Q\left(\frac{1}{i} \frac{d}{d z}\right) \varphi_{u}(z)$ around the origin is constant and so it belongs to $\widetilde{\mathscr{O}}(\boldsymbol{D})$. Hence, (5.12) holds in $\boldsymbol{D}$.

Consequently, it follows from (5.6), (5.8) and (5.11) that

$$
\check{P}\left(\frac{1}{i} \frac{d}{d x}\right) l_{2} K u=\sqrt{2 \pi} \check{S}_{u}\left(\frac{1}{i} \frac{d}{d x}\right) \delta \quad \text { in } D .
$$

By taking the Fourier transform of both sides, it can be shown that

$$
P(-\lambda) \widehat{K u}(\lambda)=\sqrt{2 \pi} S_{u}(-\lambda), \quad \lambda \in \boldsymbol{R} .
$$

Noting that $K u$ is real, we see from (2.13), (5.5), (5.7) and (5.16) that $S_{u} \in Z_{\Delta}$ and $u=\widehat{S_{u} \Delta}$. By (2.27), this implies

$$
U_{2} u=S_{u} \text {. }
$$

Since $S_{u}$ is an entire function of infra-exponential type, it follows from Corollary 2.2, (4.6) and (5.17) that $u \in \partial K(0)$. Thus, we have proved (5.2).

(Q.E.D.)

Combining the result in this section with Theorems 2.1 and 4.2 , we obtain 
THEOREM 5.2. The following conditions (i), (ii) and (iii) are equivalent:

( i ) The process $\boldsymbol{X}$ has the Markovian property;

(ii) The operator $K$ has the local property;

(iii) The symbol $\check{h}^{-1}$ of $K$ is an entire function of infra-exponential type. In concluding this section, we shall give three remarks.

REMARK 5.1. In $\S 4$, using two methods, we have proved Theorem 4.2, which states that (ii) and (iii) in Theorem 5.2 are equivalent. The one of these methods has required Lemma 4.3. When (iii) in Theorem 5.2 holds, our proof in this section gives another one of Lemma 4.3. Moreover, by (5.6), (5.8), (5.16) and (5.17), we have

COROLlary 5.1. If the process $\boldsymbol{X}$ has the Markovian property, then there uniquely exists a system $\left\{S_{u} ; u \in \partial K(0)\right\}$ of entire functions of infraexponential type such that

(i ) $S_{u}$ is given by (5.13) and (5.14),

(ii) $Q\left(\frac{1}{i} \frac{d}{d x}\right) l_{1} u=2 \pi \check{S}_{u}\left(\frac{1}{i} \frac{d}{d x}\right) \delta \quad$ in $D$,

(iii) $U_{2} u=S_{u}$ and $\partial Z_{4}(0)=\left\{S_{u} ; u \in \partial K(0)\right\}$.

REMARK 5.2. Let $A$ be a natural isomorphism from $K$ onto $K^{\prime}$ :

$$
\langle A u, v\rangle=(u, v)_{K}, \quad \text { for any } u, v \in \boldsymbol{K} .
$$

If we use the terminology of L. Schwartz [24], (2.27) implies that $K$ becomes a real Hilbert subspace of $\widetilde{L^{\prime}}$. Let $N$ be the kernel associated with $K$ :

$$
N=A^{-1} \circ i^{\prime}
$$

where $i$ is a natural injection from $K$ into $\widetilde{L^{1}}$ and $i^{\prime}$ is its dual. Since the image of $N$ is dense in $\boldsymbol{K}, \boldsymbol{A}$ is uniquely determined by $N$. Taking this into account, we say that $A$ is a generator of the process $X$.

REMARK 5.3. We suppose that $\boldsymbol{X}$ has the Markovian property. We set $\mathscr{P}_{*}^{r}=\left\{f \in \mathscr{P}_{*} ; f(\boldsymbol{R}) \subset \boldsymbol{R}\right\}$ (Definition 3.4). Then, we can prove that $\mathscr{P}_{*}^{r}$ is included in $K$ and the injection is continuous with dense range in $K$, and that the diagram

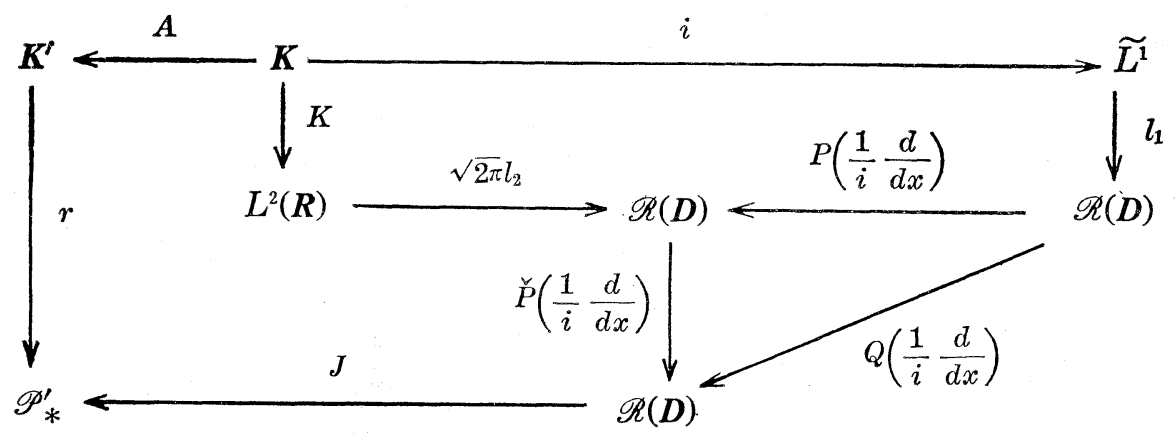


commutes, where $r$ is a restriction mapping and $J$ is a bijective mapping defined in (3.11).

\section{§ 6. Hyperprocesses and germ space (I)}

As stated in $\S 1$, a class of $\boldsymbol{X}$ which K. Urbanik [26] has considered satisfies the properties (i) $\boldsymbol{X}$ has the Markovian property, (ii) $\boldsymbol{X}$ is infinitely differentiable in the mean and (iii) $\partial \boldsymbol{M}(0)=\boldsymbol{M}_{\infty}(0)$, where $\boldsymbol{M}_{\infty}(0)$ denotes the closed linear hull of $\left\{X^{(n)}(0) ; n \in N_{*}\right\}$. On the other hand, in N. LevinsonH. P. McKean, Jr. [14] and H. Dym-H. P. McKean, Jr. [6], there is given an example of $\boldsymbol{X}$ having the properties (i), (ii) and (iv) $\partial \boldsymbol{M}(0) \supseteq \boldsymbol{M}_{\infty}(0)$ 。 These examples show that $\partial \boldsymbol{M}(0)$ cannot be always generated by $L^{2}$-derivatives of all order of $\boldsymbol{X}$ at the origin. However, we know from Definition 2.3 that any element of $\partial \boldsymbol{M}(0)$ is determined by the local information of $\boldsymbol{X}$ around the origin. "If we adjoin what kinds of local functionals from $\partial M(0) \ominus$ $\boldsymbol{M}_{\infty}(0)$ to $\boldsymbol{M}_{\infty}(0)$, can we fill out $\partial \boldsymbol{M}(0)$ ?" This is an open problem in [6].

In this section, by introducing the hyperprocess $\mu_{X}$ which has $X$ as its "boundary process", we shall view $\partial \boldsymbol{M}(0)$ and characterize it in such a form that if we look at its form, at once we can see how the elements of $\partial \boldsymbol{M}(0)$ are determined by the values of $\boldsymbol{X}$ in arbitrary small neighbourhood of the origin (Theorem 6.1 ).

Now, by taking an $L^{2}$-function $\sqrt{2 \pi}-1$ in (2.15) as one of the kernels in moving representations of $X([11])$, we have

LEMMA 6.1. There uniquely exists a Brownian motion $(B(t) ; t \in \boldsymbol{R})$ such that for any $t \in \boldsymbol{R}$

$$
\begin{gathered}
X(t)=\int_{R} \frac{1}{\sqrt{2 \pi}} E(t-s) d B(s), \\
F((-\infty, t))=\sigma(d B(s) ; s \in(-\infty, t)) .
\end{gathered}
$$

REMARK 6.1. We note that (6.2) is equivalent to (2.20). By means of Lemma 3.2 (iii), we can define a complex stochastic process $\varphi_{X}=\left(\varphi_{X}(z)\right.$; $z \in \boldsymbol{C}-\boldsymbol{R}$ ) with time parameter in $\boldsymbol{C}-\boldsymbol{R}$ by

$$
\varphi_{X}(z)=\int_{R} \sqrt{2 \pi}^{-1} \varphi_{E}(z-s) d B(s), \quad z \in C-R .
$$

Then we shall show

LEMMA 6.2. (i) $\varphi_{\boldsymbol{X}}(z)$ has the strong derivatives in $L^{2}(\Omega, F, P)$ with respect to $z(\in \boldsymbol{C}-\boldsymbol{R})$ infinitely many times,

(ii) $\underset{\varepsilon \downarrow 0}{\text { 1.i.m. }} \varphi_{X}(\cdot \pm i \varepsilon) \equiv \varphi_{X}(\cdot \pm i 0)$ exist and $\varphi_{X}(\cdot+i 0)-\varphi(\cdot-i 0)=X(\cdot)$, 
(iii) $\varphi_{X}^{(n)}(z)=\int_{R} \sqrt{2 \pi}^{-1} \varphi_{x}^{(n)}(z-s) d B(s)$, for any $n \in N_{*}$ and any $z \in C-R$.

Proof. By the isometry of stochastic integral with respect to $L^{2}$, we see that (i) and (iii) (resp. (ii)) follow from Lemma 3.2 (iii) (v) (resp. Lemma 3.2 (iii) (iv)).

(Q.E.D.)

REMARK 6.2. Applying a theorem in L. Arnold [1] to $\varphi_{X}$ by Lemma 6.2 (i), we have a version of $\varphi_{X}$ which is holomorphic in $C-R$ for almost all $\omega \in \Omega$.

Next we shall introduce the concept of hyperprocesses.

DEFINITION 6.1. A family $\mu=\left(\mu_{\omega} ; \omega \in \Omega\right)$ of elements in $\mathscr{B}(R)$ is called a hyperprocess on $(\Omega, F, P)$ if there exists a function $\varphi(z, \omega)$ such that, for any fixed $\omega, \varphi(\cdot, \omega)$ is a defining function of the hyperfunction $\mu_{\omega}$, and, for any fixed $z, \varphi\left(z,{ }^{\circ}\right)$ is $F$-measurable. Such a function $\varphi(z, \omega)$ is called a defining process of the hyperprocess $\mu$. Furthermore we say that two hyperprocesses $\mu^{(j)}=\left(\mu_{\omega}^{(j)} ; \omega \in \Omega\right)$ equal one another if $\mu_{\omega}^{(1)}=\mu_{\omega}^{(2)}$ for almost all $\omega \in \Omega$.

By virtue of Remark 6.2, we can define a hyperprocess $\mu_{X}$ with the defining process $\varphi_{X}(z)$ in $(6.3)$ :

$$
\mu_{X}=\left(\left[\varphi_{X}(z), C\right]\right) .
$$

On the other hand, it is easy to verify that for any $f \in C_{0}^{\infty}(\boldsymbol{R})$

$$
\varphi_{f X}(z)=\frac{1}{2 \pi i} \int_{R} \frac{f(t) X(t)}{t-z} d t \quad(z \in C-\boldsymbol{R})
$$

is absolutely convergent in $\boldsymbol{C}-\boldsymbol{R}$ and it has the strong derivatives in $L^{2}(\Omega, F, P)$ with respect to $z \in C-R$. Then we shall show

LEMMA 6.3. If a function $f \in C_{0}^{\infty}(\boldsymbol{R})$ is equal to one in $(a, b)$, then

$$
\text { l.i.m. }\left(\varphi_{f \backslash 0}(x+i \varepsilon)-\varphi_{f X}(x-i \varepsilon)\right)=X(x), \quad \text { for any } x \in(a, b) \text {. }
$$

Proof. It follows from (6.1) and (6.5) that for any $x \in(a, b)$ and any $\varepsilon>0$

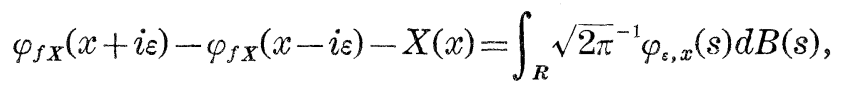

where $\varphi_{\varepsilon, x}$ is an $L^{2}$-function defined by

$$
\varphi_{\varepsilon, x}(s)=\int_{R}(f(x+\varepsilon t) E(x+\varepsilon t-s)-f(x) E(x-s)) \frac{1}{\pi\left(1+t^{2}\right)} d t .
$$

Since

$$
\left\|\varphi_{s, x}\right\|_{2}^{2} \leqslant 2\|E\|_{2}^{2} \int_{R} \frac{|f(x+\varepsilon t)-f(x)|^{2}}{\pi\left(1+t^{2}\right)} d t+2 \int_{R} \frac{\|E(\varepsilon t+\cdot)-E(\cdot)\|_{2}^{2}}{\pi\left(1+t^{2}\right)} d t,
$$


it follows that l.i.m. $\varphi_{a, x}=0$ for any $x \in(a, b)$. Therefore, we obtain Lemma 6.3 using the isometry of stochastic integral with respect to $L^{2}$. $\quad$ (Q.E.D.)

Noting that by (3.7) and Definition 6.1 we can operate any local operator in $\mathscr{R}$ to any hyperprocess, we shall show

LEMMA 6.4. Let $S(\cdot)=\sum_{n=0}^{\infty} a_{n}(i \cdot)^{n}$ be any entire function of infraexponential type. Then we have

( i ) $S\left(\frac{1}{i} \frac{d}{d x}\right) \mu_{X}=\left(\left[S\left(\frac{1}{i} \frac{d}{d z}\right) \varphi_{X}(z), C\right]\right)$,

(ii) $S\left(\frac{1}{i} \frac{d}{d z}\right) \varphi_{X}(z)=\sum_{n=0}^{\infty} a_{n} \varphi_{X}^{(n)}(z)$ is convergent in $L^{2}(\Omega, F, P)$ uniformly in $\{z \in \boldsymbol{C}-\boldsymbol{R} ;|\operatorname{Im} z| \geq \delta\}$ for any $\delta>0$,

(iii) $S\left(\frac{1}{i} \frac{d}{d z}\right) \varphi_{X}(z)=\int_{R} \sqrt{2 \pi}^{-1} S\left(\frac{1}{i} \frac{d}{d z}\right) \varphi_{E}(z-s) d B(s)$, for any $z \in \boldsymbol{C}-\boldsymbol{R}$. For the proof of Lemma 6.4, by Lemmas 3.2 (iii) and 6.2 (i) (iii), it suffices to prove

LEMMA 6.5. Let $S$ be the same as in Lemma 6.4. For any $u=\tilde{f} \in \widetilde{L}^{2}$ and any $\delta>0$

$$
\lim _{N \rightarrow \infty} \sup _{|y| \geq^{\delta}}\left\|S\left(\frac{1}{i} \frac{d}{d z}\right) \varphi_{u}(\cdot+i y)-\sum_{n=0}^{N} a_{n} \varphi_{u}^{(n)}(\cdot+i y)\right\|_{L^{2}}=0 .
$$

Proof. It follows from Lemma 3.4 (ii) that for any $N \in N$ and any $z \in C^{+}$

$S\left(\frac{1}{i} \frac{d}{d z}\right) \varphi_{u}(z)-\sum_{n=0}^{N} a_{n} \varphi_{u}^{(n)}(z)=\frac{1}{2 \pi} \int_{0}^{\infty} e^{i x t} e^{-y t}\left(\sum_{n=N+1}^{\infty} a_{n}(i t)^{n}\right) f(t) d t, \quad z=x+i y$

Since by (3.6) there exists some constant $c_{\tilde{\delta}}>0$ such that, for any $t>0$ and $y \geq \delta$, the estimate $e^{-y t} \sum_{n=0}^{\infty}\left|a_{n}\right||t|^{n} \leqslant c_{\delta}$ holds, we find from Plancherel's theorem and Lebesgue's dominated convergence theorem that the assertion for $y \geq \delta$ in Lemma 6.4 holds. Similarly, we have the one for $y \leqslant-\delta . \quad$ (Q.E.D.)

For the purpose of stating main Theorem 6.1 in this section, we define a subspace $L_{\Delta}$ of local operators in $\mathscr{R}$ as follows:

(6.6) $L_{\Delta}=$ the set of all entire functions $S$ of infra-exponential type such that $S(\cdot) \in Z_{\Delta}$.

With the aid of Lemma 4.3 and (4.6), it follows from Propositions 2.1, 2.7, Corollaries 2.1, 2.2, (2.6), (2.27) and (6.6) that there uniquely exists a bijective linear operator $U$ from $\partial M(0)$ onto $L_{\Delta}$ such that for any $Y \in \partial M(0)$ and any $t \in \boldsymbol{R}$ 


$$
(Y, X(t))_{M}=\widehat{U Y \cdot \Delta}(t)
$$

Furthermore we shall prepare

LEMMA 6.6. For any $S \in L_{\Delta}$ and any $x \in \boldsymbol{R}$

$$
\underset{e \downarrow 0}{\lim .} S\left(\frac{1}{i} \frac{d}{d z}\right) \varphi_{X}(x \pm i \varepsilon) \quad \text { exist in } L^{2}(\Omega, F, P)
$$

Proof. Since $S \in L_{\Delta}$, it follows from (2.13), (2.15) and (6.6) that $S(\cdot) \hat{E} \in L^{2}$. Noting this, we see from Lemma 3.4 (ii) that for any $z \in \boldsymbol{C}-\boldsymbol{R} S\left(\frac{1}{i} \frac{d}{d z}\right) \varphi_{E}(z)$

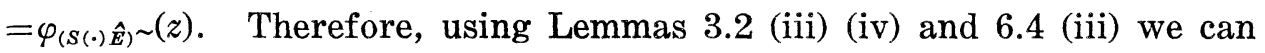
prove Lemma 6.6.

(Q.E.D.)

From (6.4) and Lemma 6.6, it follows that for any $S \in \boldsymbol{L}_{\Delta}$ and any $x \in \boldsymbol{R}$ there exists a positive sequence $\left(\varepsilon_{n}\right)_{n=1}^{\infty}$ tending to zero for which the limits $\lim _{n \rightarrow \infty} S\left(\frac{1}{i} \frac{d}{d z}\right) \varphi_{X}\left(x \pm i \varepsilon_{n}\right)$ exist almost surely and their difference is independent of the choice of another defining processes of the hyperprocess $\mu_{X}$ and of another positive sequences tending to zero if we choose appropriate subsequences. Therefore, we can give

Definition 6.2. For any $S \in \boldsymbol{L}_{\boldsymbol{A}}$ and any $x \in \boldsymbol{R}$, we set

$$
S\left(\frac{1}{i} \frac{d}{d x}\right) \mu_{X}(x)=\lim _{n \rightarrow \infty} S\left(\frac{1}{i} \frac{d}{d z}\right) \varphi_{X}\left(x+i \varepsilon_{n}\right)-\lim _{n \rightarrow \infty} S\left(\frac{1}{i} \frac{d}{d z}\right) \varphi_{X}\left(x-i \varepsilon_{n}\right)
$$

Now, after above preparations, we are in a position to prove

THEOREM 6.1. (i) For any $Y \in \partial M(0)$, set $S=U Y$. Then

$$
Y=S\left(\frac{1}{i} \frac{d}{d x}\right) \mu_{X}(0)
$$

(ii) For any $S \in L_{\Delta}$, set $Y=S\left(\frac{1}{i} \frac{d}{d x}\right) \mu_{X}(0)$. Then

$$
Y \in \partial M(0) \text { and } U Y=S
$$

Proof. (i) We define $f=K U_{1}^{-1} Y\left(\in L^{2}(R)\right)$. It follows from Propositions 2.1, 2.5, Lemma 2.15, (6.1) and (6.7) that

$$
Y=\int_{R} f(s) d B(s) \text { and } f=\sqrt{2 \pi}^{-1}(S(\cdot) \check{h})^{\wedge}
$$

Therefore, using Lemmas 3.2 (iii) (iv) and 6.4 (iii), we see that 


$$
S\left(\frac{1}{i} \frac{d}{d x}\right) \mu_{X}(0)=\int_{R} \sqrt{2 \pi}^{-1}\left(S(\cdot) \check{h}^{\wedge}(s) d B(s)=Y .\right.
$$

This completes the proof of (i). Next, we shall show (ii). By Lemma 6.6 and Definition 6.2, it is clear that $Y \in M$. Fix any $\varepsilon>0$. Furthermore, take a function $f \in C_{0}^{\infty}(R)$ such that $f=1$ in $\left(-\frac{\varepsilon}{2}, \frac{\varepsilon}{2}\right)$ and $f=0$ in $(-\infty,-\varepsilon) \cup$ $(\varepsilon, \infty)$. Then, by virtue of Lemmas 6.2 (ii), 6.3 and $(6.8)$

$$
Y=\lim _{n \rightarrow \infty}\left\{S\left(\frac{1}{i} \frac{d}{d z}\right) \varphi_{f X}\left(i \varepsilon_{n}\right)-S\left(\frac{1}{i} \frac{d}{d z}\right) \varphi_{f X}\left(-i \varepsilon_{n}\right)\right\},
$$

where $\left(\varepsilon_{n}\right)_{n=1}^{\infty}$ is some positive sequence tending to zero. On the other hand, by (6.5), $S\left(\frac{1}{i} \frac{d}{d z}\right) \varphi_{f X}\left( \pm i \varepsilon_{n}\right)$ are $\boldsymbol{F}((-\varepsilon, \varepsilon))$-measurable. Therefore, we see that $Y$ is $\boldsymbol{F}((-\varepsilon, \varepsilon))$-measurable and hence $\partial \boldsymbol{F}(0)$-measurable since $\varepsilon$ is arbitrary. By Lemma 2.1 (ii), $Y \in \partial M(0)$. Moreover, it follows from (6.10) that $(U Y)\left(\frac{1}{i} \frac{d}{d x}\right) \mu_{X}(0)=S\left(\frac{1}{i} \frac{d}{d x}\right) \mu_{X}(0)$. This implies that $U Y=S$. Thus we have proved (ii).

REMARK 6.3. Since the relation (6.7) between $Y$ and $U Y$ is given through the integration, it is not clear how $Y(\in \partial \boldsymbol{M}(0))$ is determied by the local information of $\boldsymbol{X}$ at the origin by means of $U Y\left(\in \boldsymbol{L}_{A}\right)$. But, Theorem 6.1 disposes of its mechanism.

Finally, in case $\boldsymbol{X}$ has the Markovian property, we consider the problem of prediction by means of the past and give a concrete prediction formula as an application of Corollary 5.1 and Theorem 6.1.

THEOREM 6.2. If the process $\boldsymbol{X}$ has the Markovian property, then there uniquely exists a system $\left\{S_{t} ; t>0\right\}$ of elements in $\boldsymbol{L}_{\Delta}$ such that

$$
E\left(X(t) \mid \boldsymbol{F}^{-}(0)\right)=S_{t}\left(\frac{1}{i} \frac{d}{d x}\right) \mu_{X}(0) .
$$

Furthermore, each $S_{t}$ is given by

$$
S_{t}(\cdot)=\sum_{n=0}^{\infty} a_{n}(t)(i \cdot)^{n} \quad \text { and } \quad a_{n}(t)=\frac{1}{2 \pi n !} \oint z^{n} \varphi_{t}(z) d z,
$$

where the integral in (6.12) is taken on a sufficiently small sphere with the centre 0 (the origin of $\boldsymbol{C}$ ), and its value is independent of such a sphere and then $\varphi_{t}$ is an element of $\mathcal{O}(\boldsymbol{C}-\{0\})$ defined by

$$
\varphi_{t}(z)=\frac{1}{2 \pi i} \check{P}\left(\frac{1}{i} \frac{d}{d z}\right) \int_{-\infty}^{0} \frac{E(t-s)}{s-z} d s, \quad z \in \boldsymbol{C}-\boldsymbol{R} .
$$


Proof. Fix any $t>0$ and then set $u_{t}=P_{K^{-(0)}} k(\cdot-t)$. Since by Proposition 2.2 the Markovian property of $\boldsymbol{X}$ yields $u_{t} \in \partial K(0)$, we see from Corollary 5.1 that it is possible to define $S_{t}$ in $L_{\Delta}$ by

$$
S_{t}=S_{u_{t}} \text {. }
$$

Therefore, for the proof of Theoren 6.2, by virtue of Corollary 5.1 (iii), (5.13) and Theorem 6.1, we have only to show that $Q\left(\frac{1}{i} \frac{d}{d z}\right) \varphi_{u_{t}}(z)=\varphi_{t}(z)$ in (6.13) for any $z \in \boldsymbol{C}-\boldsymbol{R}$. But, this follows immediately from (5.14) if we note that $K u_{t}=\frac{1}{\sqrt{2 \pi}} \chi_{(-\infty, 0)}(\cdot) \cdot E(t-\cdot)$, which is proved by Proposition 2.5 and Lemma 2.12 (ii).

REMARK 6.4. It follows from (6.12) and (6.13) that local operators $S_{t}$ in (6.11) can be given directly by means of $P$ and $E$. However this formula needs a calculation on the complex domain. In the next section, under an additional assumption on $P$, we shall obtain a more concrete prediction formula by calculating $a_{n}(t)$, which can be computed only on the real domain. In particular when $P$ is a polynomial of order $N$, it is seen immediately from the precise prediction formula that each $S_{t}$ is a polynomial of at most order $N-1$. Therefore, in such a case, it will be shown in $\S 7$ and $\S 9$ that (6.11) can be transformed into

$$
E\left(X(t) \mid \boldsymbol{F}^{-}(0)\right)=\sum_{n=1}^{N-1} a_{n}(t) X^{(n)}(0),
$$

which is well-known ([5]).

REMARK 6.5. We assume that $\boldsymbol{X}$ has the Markovian property. Then, by using the local property of the operato $K$, we can prove that

$$
\sigma(d B(u) ; s \leqslant u \leqslant t) \subseteq \boldsymbol{F}((s, t)), \quad \text { for any } s, t \in \boldsymbol{R}, s<t .
$$

\section{§ 7. Prediction operator using the germ field}

We have seen in Theorem 6.2 that the predictors $E\left(X(t) \mid \boldsymbol{F}^{-}(0)\right)$ of $X(t)$ $(t>0)$ conditional on the knowledge of the past are given as the local functionals of $\boldsymbol{X}$ at the origin by means of the system $\left\{S_{t} ; t>0\right\}$ in $Z_{\Delta}$. As noted in $\S 6$, however, it is not clear how $S_{t}$ depends upon a parameter $t$ if we would see its dependency in the real domain. The aim in this section is to examine the $t$-dependency of $S_{t}$ and obtain its concrete form in the real domain. In doing so, we shall introduce a concept of a prediction operator using the germ field.

Throughout this section we shall assume that $X$ has the Markovian 
property. Therefore, by Theorem 5.2, there exists an entire function $P$ of infra-exponential type such that

$$
\check{h}^{-1}=P \text {. }
$$

We then define an entire function $Q$ of the same type as $P$ by

$$
Q=P \cdot \check{P} .
$$

It follows from (2.13), (7.1) and (7.2) that

$$
\check{\Delta}^{-1}=Q \text {. }
$$

Therefore, by (2.9), (2.15), (3.7), (3.13), (3.15), (7.1) and (7.3), we have

$$
P\left(\frac{1}{i} \frac{d}{d x}\right) l_{2} E=2 \pi \delta, \quad \check{P}\left(\frac{1}{i} \frac{d}{d x}\right) l_{2} \check{E}=2 \pi \delta \quad \text { and } \quad Q\left(\frac{1}{i} \frac{d}{d x}\right) l_{1} k=2 \pi \delta \text {. }
$$

REMARK 7.1. In $\S 5$ we have used (7.4) implicitly. (7.4) implies that (1.10) holds in the sense of Fourier hyperfunctions in a general case.

For any open set $D$ in $R$, we denote by $\mathscr{A}(D)$ the set of all real analytic functions in $D$. Fix any open set $D$ in $\boldsymbol{R}$ and any $f \in \mathscr{A}(D)$. Since there exists a set $V \in \mathscr{V}(D)$ such that $f \in \mathcal{O}(V \cap C)$, we have a hyperfunction $f \cdot 1(\in \mathscr{B}(D))$ defined by $f \cdot 1=[\varepsilon(z) f(z), V]$, where $\varepsilon(z)=1$ in $C^{+}$and $\varepsilon(z)=0$ in $C^{-}$. Then, the correspondence $f \rightarrow f \cdot 1$ induces an isomorphism from $\mathscr{A}(D)$ into $\mathscr{B}(D)$. Hereafter, we consider $\mathscr{A}(D)$ as canonically imbedded in $\mathscr{B}(D)$. In such a sense, we can define for any $\mu \in \mathscr{B}(\boldsymbol{R}) \operatorname{singsupp} \mu$ by the minimal closed set in $R$ outside of which $\mu$ is real analytic.

At first, we shall give a characterization for the real analyticity in the positive axis of $E$ from an operator-theoretical point of view. When $P$ is polynomial, it is well-known that thedi fferential operator $P\left(\frac{1}{i} \frac{d}{d x}\right)$ is elliptic in the sense of $L$. Schwartz's distributions and hence, by (7.4), $E$ is real analytic in $(0, \infty)$. In order to treat a more general case, following $\mathrm{T}$. Kawai [12], we shall give the definitions of hyperbolicity and ellipticity of local operators in the sense of M. Sato's hyperfuctions.

Definition 7.1. A local operator $S\left(\frac{1}{i} \frac{d}{d x}\right)$ in $\mathscr{R}$ is said to be hyperbolic in the positive direction if there exists some $E \in \mathscr{B}(\boldsymbol{R})$ such that

$$
S\left(\frac{1}{i} \frac{d}{d x}\right) E=\delta \quad \text { in } R \text { and } \operatorname{supp} E \subset[0, \infty) .
$$

The hyperbolicity in the negative direction is also defined in the same way. Since by (2.16) and Lemma 3.3 


$$
l_{2} E=0 \text { in }[-\infty, 0) \text { and } l_{2} \check{E}=0 \text { in }(0, \infty],
$$

we see from (7.4) that

Proposition 7.1. Two local operators $P\left(\frac{1}{i} \frac{d}{d x}\right)$ and $\check{P}\left(\frac{1}{i} \frac{d}{d x}\right)$ are are hyperbolic in the positive and negative direction, respectively.

DEFINITION 7.2. A local operator $S\left(\frac{1}{i} \frac{d}{d x}\right)$ in $\mathscr{R}$ is said to be elliptic if any hyperfunction solution $u$ of $S\left(\frac{1}{i} \frac{d}{d x}\right) u=f$ is real analytic where $f$ is real analytic.

Since for any local operator $S\left(\frac{1}{i} \frac{d}{d x}\right)$ in $\mathscr{R}$ and any open set $D$ in $\boldsymbol{R}$

$$
S\left(\frac{1}{i} \frac{d}{d x}\right) \mathscr{A}(D) \subset \mathscr{A}(D)
$$

we get

LEM MA 7.1. The ellipticity of three local operators $P\left(\frac{1}{i} \frac{d}{d x}\right), \check{P}\left(\frac{1}{i} \frac{d}{d x}\right)$ and $Q\left(\frac{1}{i} \frac{d}{d x}\right)$ is equivalent each other.

By combining Theorem 4.1.2 in [12], the proof therein and Theorem 4.1.9' in [12], we obtain

LEMMA 7.2. Let $S\left(\frac{1}{i} \frac{d}{d x}\right)$ be any local operator in $\mathscr{R}$ and $V_{S}$ the set of zero points of $S(\cdot)$. Then the conditions (i) and (ii) are equivalent:

(i) There exists a constant $c>0$ such that if $\xi$ belongs to $\boldsymbol{R}$ and $|\xi|$ is sufficiently large, then the distance from $\xi$ to $V_{S} \geq c|\xi|$;

(ii) There exists a hyperfunction $\mu \in \mathscr{B}(\boldsymbol{R})$ with singsupp $\mu=\{0\}$ and an entire function $f \in \mathcal{O}(C)$ such that $S\left(\frac{1}{i} \frac{d}{d x}\right) \mu=\delta+f$.

Furthermore, if (i) (and so (ii)) holds, then $S\left(\frac{1}{i} \frac{d}{d x}\right)$ is elliptic.

Now, after above preparations, we shall show

THEOREM 7.1. The following (i) (vi) are equivalent:

(i ) $P\left(\frac{1}{i} \frac{d}{d x}\right)$ is elliptic;

(ii) $Q\left(\frac{1}{i} \frac{d}{d x}\right)$ is elliptic;

(iii) $E$ is real analytic in $(0, \infty)$;

(iv) $k$ is real analytic except at the origin; 
(v) $P$ satisfies Lemma 7.2 (i) for $S=P$;

(vi) $Q$ satisfies Lemma 7.2 (i) for $S=Q$.

Proof. It follows from (7.4), (7.5) and Lemma 7.1 that (i) is equivalent to (ii) and (i) (resp. (ii)) implies (iii) (resp. (iv)). Furthermore, by (7.4) and Lemma 7.2, (v) (resp. (vi)) implies (iii) (resp. (iv)). Assume that (iii) holds. Then, since by (7.5) singsupp $l_{2} E=\{0\}$, (v) follows from (7.4) and Lemma 7.2. Similarly, (iv) implies (vi).

(Q.E.D.)

Next we denote by $O(\{0\})$ the space of all holomorphic functions around the origin with the inductive limit topology. Let $P$ be expanded into a Taylor series :

$$
P(\cdot)=\sum_{n=0}^{\infty} c_{n}(-i \cdot)^{n} \text { and } \quad \lim _{n \rightarrow \infty} \sqrt[n]{\left|c_{n}\right| n !}=0 .
$$

We note that by (2.13) and (7.1) $c_{n}\left(n \in N_{*}\right)$ is real. Then, for any $z \in C$ and any $\varphi \in \mathcal{O}(\{0\})$, we define a function $P(z, \varphi)$ by

$$
P(z, \varphi)=-\frac{1}{2 \pi} \sum_{n=0}^{\infty}\left(\sum_{k=0}^{\infty} c_{n+k+1} \varphi^{(k)}(0)\right)(-i z)^{n} .
$$

This is well-defined by (7.6) and the fact that there exist constants $M_{j}>0$ $(j=1,2)$ and for any $k \in N_{*}$ the estimate $\left|\varphi^{(k)}(0)\right| \leqslant M_{1} M_{2}^{k} k$ ! holds. Moreover it can be proved that

$$
P(z, \cdot) \in \mathcal{O}(\{0\})^{\prime} \quad \text { for any fixed } \mathrm{z} \in C,
$$

(7.9) $P(\cdot, \varphi)$ is an entire function of infra-exponential type for any fixed $\varphi \in \mathcal{O}(\{0\})$.

Now we are in a position to prove

THEOREM 7.2. If $E$ is real analytic in $(0, \infty)$,

$$
S_{t}(z)=P(z, E(t-\cdot)), \quad \text { for any } t \in(0, \infty) \text { and any } z \in C \text {. }
$$
by

Proof. Fix any $t>0$ and then define a function $e \in L^{2}(R) \cap \mathscr{A}((-\infty, t))$

$$
e(\cdot)=E(t-\cdot) \quad \text { in } R
$$

This is possible by our assumption. Then, there exists some $\delta \in\left(0, \frac{t}{2}\right)$ $\cap(0,1)$ such that

$$
e \in \mathcal{O}(|z|<2 \delta) \text {. }
$$

At first, we calculate $\varphi_{t}$ in (6.13). We set $D_{\delta}=\{z \in C ;|z|<\delta\}$. For any $z \in(\boldsymbol{C}-\boldsymbol{R}) \cap D_{\delta}$ 


$$
\int_{-\infty}^{0} \frac{E(t-s)}{s-z} d s=\int_{-\infty}^{-\delta} \frac{e(s)}{s-z} d s+\int_{-\delta}^{0} \frac{e(s)-e(z)}{s-z} d s+e(z) \int_{-\delta}^{0} \frac{1}{s-z} d s .
$$

Since the first term of the right hand side belongs to $\mathcal{O}(\{z \in C ; \operatorname{Re} z>-\delta\})$ and the second term belongs to $\mathcal{O}\left(D_{\delta}\right)$ by (7.11), it follows that there exists some $\psi_{1} \in \mathcal{O}\left(D_{\delta}\right)$ and for any $z \in(C-R) \cap D_{\delta}$

$$
\varphi_{t}(z)=\frac{1}{2 \pi i} \check{P}\left(\frac{1}{i} \frac{d}{d z}\right)\left(e(z) \int_{-\delta}^{0} \frac{1}{s-z} d s\right)+\psi_{1}(z) .
$$

We define a function $\pi \in \mathcal{O}(\boldsymbol{C}-\boldsymbol{R})$ by

$$
\pi(z)=\left\{\begin{array}{l}
1+\frac{1}{2 \pi i} \log (-z), \quad z \in C^{+}, \\
\frac{1}{2 \pi i} \log (-z), \quad z \in C^{-},
\end{array}\right.
$$

where $\log$ is a logarithm function with the principal branch $C-[0, \infty)$. Since $\log (-z-\delta) \in \mathcal{O}\left(D_{\delta}\right)$ and for any $z \in \boldsymbol{C}-\boldsymbol{R}$

$$
\frac{1}{2 \pi i} \int_{-\delta}^{0} \frac{1}{s-z} d s=\pi(z)-\frac{1}{2 \pi i} \log (-z-\delta),
$$

it follows from (7.11) and (7.12) that there exists some $\psi_{2} \in \mathcal{O}\left(D_{\delta}\right)$ and for any $z \in(\boldsymbol{C}-\boldsymbol{R}) \cap D_{\text {。 }}$

$$
\varphi_{t}(z)=\check{P}\left(\frac{1}{i} \frac{d}{d z}\right)(e(z) \pi(z))+\psi_{2}(z) .
$$

By (7.6), for any $z \in(C-R) \cap D_{\delta}$,

$$
\check{P}\left(\frac{1}{i} \frac{d}{d z}\right)(e(z) \pi(z))=\sum_{k=0}^{\infty}\left(\sum_{n=k}^{\infty}\left(\begin{array}{l}
n \\
k
\end{array}\right) c_{n} e^{(n-k)}(z)\right) \pi^{(k)}(z) .
$$

On the other hand, it can be shown from (7.4), (7.10) and (7.11) that

$$
\sum_{n=0}^{\infty} c_{n} e^{(n)}(z)=0 \quad \text { for any } z \in D_{\delta} .
$$

Therefore, noting that for any $k \in N$ and any $z \in C-R \pi^{(k)}(z)=\frac{1}{2 \pi i}$ $\frac{(-1)^{k-1}(k-1) !}{z^{k}}$ by (7.13), we see from (7.14), (7.15) and (7.16) that for any $z \in(\boldsymbol{C}-\boldsymbol{R}) \cap D_{\delta}$

$$
\varphi_{t}(z)=\frac{1}{2 \pi i} \sum_{k=1}^{\infty}\left(\sum_{n=k}^{\infty}\left(\begin{array}{l}
n \\
k
\end{array}\right) c_{n} e^{(n-k)}(z)\right) \frac{(-1)^{k-1}(k-1) !}{z^{k}}+\psi_{2}(z) .
$$


Next we calculate $a_{n}(t)$ in (6.12). Since by (7.11) there exist constants $M_{j}>0(j=1,2)$ such that for any $n \in N_{*}$ and any $z \in D_{\delta}$

$$
\left|e^{(n)}(z)\right| \leqslant M_{1} M_{2}^{n} n !
$$

we have, for any $z \in D_{\dot{\delta}}-\{0\}$,

$$
\begin{aligned}
\sum_{k=1}^{\infty}\left(\sum_{n=k}^{\infty}\left(\begin{array}{l}
n \\
k
\end{array}\right)\left|c_{n}\right|\left|e^{(n-k)}(z)\right|\right) \frac{(k-1) !}{|z|^{k}} & \leqslant M_{1} \sum_{n=1}^{\infty}\left(M_{2}^{\vee} \frac{1}{|z|}\right)^{n}\left|c_{n}\right|\left(\sum_{k=1}^{n}\left(\begin{array}{l}
n \\
k
\end{array}\right)(n-k) !(k-1) !\right) \\
& \leqslant M_{1} \sum_{n=1}^{\infty}\left(M_{2} \vee \frac{1}{|z|}\right)^{n}\left|c_{n}\right|(n+1) !
\end{aligned}
$$

Therefore, we see from (7.6) that the first term in (7.17) is absolutely uniform convergent on any compact subset of $D_{\bar{\delta}}-\{0\}$. Since $\varphi_{t} \in \mathcal{O}(C-\{0\})$, $e \in \mathcal{O}\left(D_{\delta}\right)$ and $\psi_{2} \in \mathcal{O}\left(D_{\delta}\right)$, this implies that for any $n \in N_{*}$

$$
\begin{aligned}
\oint z^{n} \varphi_{t}(z) d z & =\frac{1}{2 \pi i} \sum_{k=1}^{\infty}(-1)^{k-1}(k-1) !\left(\sum_{m=k}^{\infty}\left(\begin{array}{c}
m \\
k
\end{array}\right) c_{m} \oint e^{(m-k)}(z) z^{n-k} d z\right) \\
& =\sum_{k=n+1}^{\infty}(-1)^{k-1}(k-1) !\left(\sum_{m=k}^{\infty}\left(\begin{array}{c}
m \\
k
\end{array}\right) c_{m} \frac{e^{(m-n-1)}(0)}{(k-n-1) !}\right) .
\end{aligned}
$$

In above calculation, we have used

$$
\frac{1}{2 \pi i} \oint e^{(m-k)}(z) z^{n-k} d z=\left\{\begin{array}{cl}
0, & \text { for } 1 \leqslant k \leqslant n, \\
\frac{e^{(m-n-1)}(0)}{(k-n-1) !}, & \text { for } n+1 \leqslant k \leqslant m .
\end{array}\right.
$$

Furthermore, since (7.19) is absolutely convergent by (7.18) and (7.20), for any $n \in N_{*}$,

$$
\begin{aligned}
a_{n}(t) & =-\frac{1}{2 \pi} \sum_{m=n+1}^{\infty} c_{m} e^{(m-n-1)}(0)\left(\sum_{k=n+1}^{m}(-1)^{k-1}\left(\begin{array}{c}
k-1 \\
n
\end{array}\right)\left(\begin{array}{c}
m \\
k
\end{array}\right)\right) \\
& =-\frac{1}{2 \pi} \sum_{m=0}^{\infty} c_{n+m+1} e^{(m)}(0)\left(\sum_{k=0}^{m}(-1)^{n+k}\left(\begin{array}{c}
n+k \\
n
\end{array}\right)\left(\begin{array}{c}
n+m+1 \\
n+k+1
\end{array}\right)\right) .
\end{aligned}
$$

We claim that for any $m, n \in N_{*}$

$$
\sum_{k=0}^{m}(-1)^{k}\left(\begin{array}{c}
n+k \\
n
\end{array}\right)\left(\begin{array}{c}
n+m+1 \\
n+k+1
\end{array}\right)=1
$$

We prove this by the method of mathematical induction on $m$ as follows: When $m$ is zero, (7.22) is clear. Suppose that (7.22) holds for $m=m_{0}$. Then

$$
\sum_{k=0}^{m_{0}+1}(-1)^{k}\left(\begin{array}{c}
n+k \\
n
\end{array}\right)\left(\begin{array}{c}
n+m_{0}+2 \\
n+k+1
\end{array}\right)=\sum_{k=0}^{m_{0}}(-1)^{k}\left(\begin{array}{c}
n+k \\
n
\end{array}\right)\left\{\left(\begin{array}{c}
n+m_{0}+1 \\
n+k+1
\end{array}\right)+\left(\begin{array}{c}
n+m_{0}+1 \\
n+k
\end{array}\right)\right\}
$$




$$
\begin{aligned}
& +(-1)^{m_{0}+1}\left(\begin{array}{c}
n+m_{0}+1 \\
n
\end{array}\right) \\
= & 1+\left(\begin{array}{c}
n+m_{0}+1 \\
n
\end{array}\right)\left\{\sum_{k=0}^{m_{0}}(-1)^{k}\left(\begin{array}{c}
m_{0}+1 \\
k
\end{array}\right)+(-1)^{m_{0}+1}\right\} \\
= & 1 .
\end{aligned}
$$

This implies that (7.22) holds for $m=m_{0}+1$ and hence (7.22) is proved. By (7.21) and (7.22),

$$
a_{n}(t)=-\frac{(-1)^{n+1}}{2 \pi} \sum_{m=0}^{\infty} c_{n+m+1} e^{(m)}(0)
$$

Thus, by (6.12), (7.7) and (7.23), we obtain Theorem 7.2.

Noting the Markovian property of $\boldsymbol{X}$ and combining Proposition 2.8, Corollary 5.1 and Theorems 6.1, 6.2 with Theorem 7.2, we have

COROLlaRY 7.1. Under the same assumption as in Theorem 7.2, for any $t>0$,

$$
\begin{gathered}
\left(P_{\partial Z_{\Delta}(0)} e^{i t \cdot)(\lambda)}=P(\lambda, E(t-\cdot)), \quad \lambda \in R,\right. \\
E(X(t) \mid \partial F(0))=P\left(\frac{1}{i} \frac{d}{d x}, E(t-\cdot)\right) \mu_{X}(0) .
\end{gathered}
$$

Furthermore, by (7.7) and Theorem 7.2,

CoRollary 7.2. If $P$ is a polynomial of degree $N$, then $S_{t}$ in (6.12) is a polynomial of at most order $N-1$ and $a_{n}(t)$ is given by

$$
a_{n}(t)=\frac{1}{2 \pi} \sum_{k=0}^{N-n-1} c_{n+k+1}(-1)^{n+k+1} E^{(k)}(t), \quad n \in\{0,1, \cdots, N-1\}
$$

REMARK 7.2. We assume that $E$ is real analytic in $(0, \infty)$. By Lemma 7.1 and Theorem 7.1, two local operators $P\left(\frac{1}{i} \frac{d}{d x}\right)$ and $\check{P}\left(\frac{1}{i} \frac{d}{d x}\right)$ are elliptic. Therefore, it can be proved from Corollary 2.1, Proposition 2.5, Lemma 2.13 (ii) and (5.8) that for any $\varepsilon>0$ and any $Y \in M^{+}(\varepsilon)$

$$
f_{Y} \equiv K U_{1}^{-1} Y \in \mathcal{O}(\{0\}) \text {. }
$$

Noting this and using the same argument as in Theorem 7.2 and Corollary 7.1 , we see that for any $\varepsilon>0$ and any $Y \in M^{+}(\varepsilon)$

$$
E(Y \mid \partial F(0))=P\left(\frac{1}{i} \frac{d}{d x}, \sqrt{2 \pi} f_{Y}\right) \mu_{X}(0) .
$$

Taking account of (7.8), (7.9), (7.25) and (7.27), it is reasonable to give 
Definition 7.3. A local operator $P\left(\frac{1}{i} \frac{d}{d x}, \varphi\right)$ (resp. a function $P(z, \varphi)$ ) is called a prediction operator (resp. a prediction function) using the germ field.

REMARK 7.3. As noted in $\S 1$, it follows from (7.8), (7.9) and (7.24) that we have given an answer to the problem of prediction by means of the germ field to which H. Dym and H. P. McKean, Jr. [6] had reduced the problem of prediction by means of a bounded segment of the past.

\section{§ 8. Formula for canonical representation kernel}

In this section we shall give a formula for the canonical representation kernel $\sqrt{2 \pi}-1$ as an application of Theorem 7.2. For that purpose, we shall assume that $\boldsymbol{X}$ has the Markovian property and $E$ is a real analytic in $(0, \infty)$.

Let $P(z, \varphi)(z \in C$ and $\varphi \in \mathcal{O}(\{0\}))$ be the prediction function using the germ field. Then we shall show

LEMMA 8.1. ( i ) $P\left(\cdot,\left\{x^{n}\right\}\right) \in \partial Z_{4}(0)$, for any $n \in N_{*}$,

(ii) $P\left(-\cdot,\left\{x^{n}\right\}\right) h(\cdot) \in H^{2+}$, for any $n \in N_{*}$,

(iii) $P\left(-\cdot,\left\{x^{n}\right\}\right) h(\cdot) \in L^{1}$, for any $n \in N$.

Proof. By (7.6) and (7.7), for any $n \in N_{*}$ and any $z \in C$,

$$
\begin{aligned}
P\left(-z,\left\{x^{n}\right\}\right) & =-\frac{n !}{2 \pi} \sum_{k=0}^{\infty} c_{n+k+1}(i z)^{k} \\
& =-\frac{n !}{2 \pi} \frac{1}{(i z)^{n+1}}\left(P(-z)-\sum_{k=0}^{n} c_{k}(i z)^{k}\right)
\end{aligned}
$$

By (2.13) and (7.1), this implies (iii) and $P\left(-\cdot,\left\{x^{n}\right\}\right) h(\cdot) \in L^{2}$ for any $n \in N_{*}$. Since $c_{k}\left(k \in N_{*}\right)$ is real, $\overline{P\left(-\cdot,\left\{x^{n}\right\}\right)}=P\left(\cdot,\left\{x^{n}\right\}\right)$ in $R$, for any $n \in N_{*}$. Therefore, by (2.13), (4.6) and (7.9), we have (i). Furthermore, since $\partial Z_{\Delta}(0) \subset Z_{\Delta}^{+}(0)$ by Lemma 2.6 (ii) and Corollary 2.2, (ii) follows from (2.20) and (i).

Now we define a sequence $\left(E_{n}\right)_{n=0}^{\infty}$ of elements in $\mathscr{A}(\boldsymbol{R}-\{0\})$ by

$$
E_{n}(t)= \begin{cases}\frac{1}{2 \pi} \sum_{k=0}^{\infty} c_{n+k+1}(-1)^{k+1} E^{(k)}(t), & t>0 \\ 0, & t \leqslant 0\end{cases}
$$

This is well-defined by (7.6), (7.10) and (7.18). Then we shall show

LEM MA 8.2. For any $n \in N_{*}$

$$
E_{n}(t)=\frac{1}{n !}\left\{P\left(-\cdot,\left\{x^{n}\right\}\right) h(\cdot)\right\}^{\wedge}(t), \quad \text { for almost all } t \in \boldsymbol{R} .
$$


Proof. Fix any $n \in N_{*}$. By (2.19), (8.2) and Lemma 8.1 (ii), Lemma 8.2 holds in the negative axis. Operating a local operator $P\left(\frac{1}{i} \frac{d}{d x},\left\{x^{n}\right\}\right)$ to $l_{2} E$, we see from Lemmas 3.4 and 8.1 (ii) that

$$
P\left(\frac{1}{i} \frac{d}{d x},\left\{x^{n}\right\}\right) l_{2} E=2 \pi l_{2}\left(\left(P\left(\cdot,\left\{x^{n}\right\}\right) \check{h}\right)^{\sim}\right)
$$

On the other hand, since $E \in \mathscr{A}((0, \infty))$, there exists a set $V \in \mathscr{V}((0, \infty))$ and a function $e \in \mathcal{O}(V \cap C)$ such that

$$
E=e \text { in }(0, \infty) \text { and } l_{2} E=[\varepsilon(z) e(z), V] \text { in }(0, \infty) .
$$

By (3.7), (8.1), 8.2) and (8.4),

$$
P\left(\frac{1}{i} \frac{d}{d x},\left\{x^{n}\right\}\right) l_{2} E=\left[\varepsilon(z) P\left(\frac{1}{i} \frac{d}{d z},\left\{x^{n}\right\}\right) e(z), V\right] \text { in }(0, \infty)
$$

and

$$
\lim _{\varepsilon \downarrow 0}\left(P\left(\frac{1}{i} \frac{d}{d z},\left\{x^{n}\right\}\right) e\right)(\cdot+i \varepsilon)=n ! E_{n}(\cdot) \quad \text { in }(0, \infty) .
$$

Therefore, using Lemma 3.2 (ii), we see from (8.3) that Lemma 8.2 holds in the positive axis.

(Q.E.D.)

After above preparations, we shall prove

THEOREM 8.1. For any $s \in(-\infty, 0)$ and any $t \in(0, \infty)$

$$
E(t-s)=\frac{1}{2 \pi} \sum_{n=0}^{\infty} E^{(n)}(t)\left(\sum_{k=0}^{\infty} c_{n+k+1}(-1)^{n+k+1} E^{(k)}(-s)\right)
$$

REMARK 8.1. By (7.6), (7.10) and (7.18), above series is absolutely convergent uniformly in $s$ (resp. $t)$ on compact set of $(-\infty, 0)($ resp. $(0, \infty))$.

Proof. Fix any $t>0$. By Lemma 2.12 (ii), Proposition 2.7, Corollary 2.2, Lemma 2.15 and (7.24), we have

$$
\chi_{(-\infty, 0)}^{(\cdot)} E(t-\cdot)=(P(\cdot, E(t-\cdot)) h(-\cdot))^{\wedge} \quad \text { in } R .
$$

Therefore, it follows from (2.15), (3.13), (3.15) and Lemma 3.4 that

$$
l_{2}\left(\chi_{(-\infty, 0)} E(t-\cdot)\right)=P\left(-\frac{1}{i} \frac{d}{d x}, E(t-\cdot)\right) l_{2} \check{E} .
$$

On the other hand, by (8.4), for any $s<0$,

$$
\lim _{\bullet \downarrow t 0}\left(P\left(-\frac{1}{i} \frac{d}{d z}, E(t-\cdot)\right) \check{e}\right)(s+i \varepsilon)=P\left(-\frac{1}{i} \frac{d}{d s}, E(t-\cdot)\right) \check{E}(s) .
$$


Hence, using Lemma 3.2 (iv), we see from (7.7) and (8.6) that, for any $s<0$,

$$
\begin{aligned}
E(t-s) & =P\left(-\frac{1}{i} \frac{d}{d s}, E(t-\cdot)\right) \check{E}(s) \\
& =-\frac{1}{2 \pi} \sum_{n=0}^{\infty}\left(\sum_{k=0}^{\infty} c_{n+k+1}(-1)^{k} E^{(k)}(t)\right)(-1)^{n} E^{(n)}(-s) \\
& =-\frac{1}{2 \pi} \sum_{n=0}^{\infty}(-1)^{n} E^{(n)}(t)\left(\sum_{k=0}^{\infty} c_{n+k+1}(-1)^{k} E^{(k)}(-s)\right) .
\end{aligned}
$$

This completes the proof of Theorem 8.1.

For the purpose of investigating the structure of the formula for $E$ in Theorem 8.1, we shall prepare several lemmas.

LEMmA 8.3. (i ) $E_{n} \in L^{2}(\boldsymbol{R}) \cap \mathscr{A}(\boldsymbol{R}-\{0\})$, for any $n \in \boldsymbol{N}_{*}$,

(ii) $E_{n} \in L^{1}$, for any $n \in N$,

(iii) $E_{0}^{(1)}=-\frac{c_{0}}{2 \pi} E$ in $(0, \infty), E_{n}^{(1)}=-E_{n-1}-\frac{c_{n}}{2 \pi} E$ in $(0, \infty)$, for any , $n \in N$,

(iv) $E_{0}(0+) \neq 0, E_{n}(0+)=0$, for any $n \in N$.

Proof. (i) and (ii) follow immediately from Lemmas 8.1 (ii) and 8.2, Lemmas 8.1 (iii) and 8.2, respectively. By (7.10), (7.16) and (8.2), we have (iii). The second part in (iv) follows from (2.19), Lemmas 8.1 (ii), 8.2 and 8.3 (ii). The first part in (iv) can be proved as follows: since $E \in L^{2}$, the limit $E_{0}(0+)$ exists by (iii). Furthermore, by (8.1) and Lemma 8.2,

$$
E_{0}=\frac{1}{2 \pi}\left(\frac{1-c_{0} h(-\cdot)}{-i}\right)^{\sim}
$$

Suppose that $E_{0}(0+)=0$. It then follows from (8.2) and (iii) that

$$
E_{0}^{(1)}=-\frac{c_{0}}{2 \pi} E \text { in the sense of L. Schwartz's distributions. By taking }
$$

the Fourier transform of both sides and noting (2.17) and (i), we see that $(i \cdot) \hat{E}_{0}=-\frac{c_{0}}{2 \pi} \hat{E}$. Therefore, by (8.7), $1-c_{0} h(-\cdot) \in L^{2}$. This is absurd since $h \in L^{2}$. Thus, we have $E_{0}(0+) \neq 0$.

(Q.E.D.)

REMARK 8.2. By Lemma 8.3 (i) (iii) (iv), $c_{0} \neq 0$.

By (7.4), (7.10), (7.16) and (8.2), we can prove

LEMMA 8.4. For any $n \in N_{*}$ and any $t \in(0, \infty)$

$$
P\left(\frac{1}{i} \frac{d}{d t}\right) E^{(n)}(t)=P\left(\frac{1}{i} \frac{d}{d t}\right) E_{n}(t)=0
$$

8.1. In this subsection we shall consider the case where $P$ is a poly- 
nomial of order $N$. By (2.13) and (7.1), we note that $N \geq 1$. In this case, it follows from (7.3) that

$$
\int_{R}|\lambda|^{2 k} \Delta(\lambda) d \lambda<\infty, \quad \text { for any } k \in\{0,1, \cdots, N-1\} .
$$

At first, we shall show

LEMMA 8.5. (i) $E^{(k)} \in L^{2}$, for any $k \in\{0,1, \cdots, N-1\}$,

(ii) $E^{(k)} \in L^{1}$ and $E^{(k)}(0+)=0$, for any $k \in\{0,1, \cdots, N-2\}$.

PRoof. It follows from (4.6) and (8.8) that

$$
(-i \cdot)^{k} \in \partial Z_{\Delta}(0), \quad \text { for any } k \in\{0,1 \cdots, N-1\} .
$$

Therefore, by (2.15),

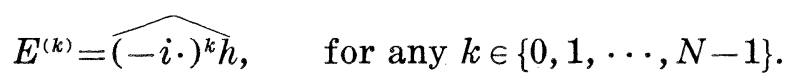

This implies (i) and the first part of (ii). Furthermore, since $\partial Z_{\Delta}(0) \subset Z_{\Delta}^{+}(0)$ by Lemma 2.6 (ii) and Corollary 2.2, the second part of (ii) follows from (2.19), (2.20), (8.9), (8.10) and the first part of (ii).

(Q.E.D.)

Using Lemmas 8.3 and 8.5 , we shall prove

LEMma 8.6. (i) $E_{n}=0$, for any $n \in\{N, N+1, \cdots\}$,

(ii) $\left\{E^{(n)} ; n=0,1, \cdots, N-1\right\}$ is linearly independent in $L^{2}(R)$,

(iii) $\left\{E_{n} ; n=0,1, \cdots, N-1\right\}$ is linearly independent in $L^{2}(\boldsymbol{R})$.

Proof. Since $c_{k}=0$ for any $k \in\{N+1, N+2, \cdots\}$, (i) holds by (8.2). Taking the inverse Fourier transform in (8.10) and noting (2.14), we have (ii). For the proof of (iii), by Lemma 8.3 (iii), we note that there exist real constant $a_{l}^{k}(k=1,2, \cdots, N-1, l=0,1, \cdots, k-1)$ and for any $n \in$ $\{k, k+1, \cdots, N-1\}$

$$
E_{n}^{(k)}=(-1)^{k} E_{n-k}+\sum_{l=0}^{k-1} a_{l}^{k} E^{(l)} \quad \text { in }(0, \infty) .
$$

Let $a_{n}(n=0,1, \cdots, N-1)$ be real constants such that

$$
\sum_{n=0}^{N-1} a_{n} E_{n}(t)=0, \quad \text { for any } t>0 .
$$

Letting $t$ tend to zero in (8.12), we see from Lemma 8.3 (iv) that $a_{0}=0$. Next, for any fixed $n_{0} \in\{1, \cdots, N-1\}$, we assume that $a_{n}=0$ for any $n \in$ $\left\{0,1, \cdots, n_{0}-1\right\}$. Then, by differentiating (8.12) $n_{0}$-times and noting (8.11),

$$
(-1)^{n_{0}} a_{n_{0}} E_{0}(t)+\sum_{n=n_{0}+1}^{N-1} a_{n}\left((-1)^{n_{0}} E_{n-n_{0}}(t)+\sum_{l=0}^{n_{0}-1} a_{l}^{n_{0}} E^{(l)}(t)\right)=0,
$$

for any $t>0$. 
Therefore, letting $t$ tend to zero, we see from Lemmas 8.3 (iv) and 8.5 (ii) that $a_{n_{0}}=0$. Thus, by the method of mathematical induction on $n$, we find that $a_{n}=0$ for any $n \in\{0,1, \cdots, N-1\}$. This completes the proof of (ii).

(Q.E.D.)

Finally in this subsection, we shall calculate the value $E_{0}(0+)$.

Proposition 8.1. $\quad E_{0}(0+)=\frac{1}{2 \pi} c_{N}(-1)^{N} E^{(N-1)}(0+)=1$.

Proof. Since $c_{k}=0$ for any $k \in\{N+1, N+2, \cdots\}$, by (8.2),

$$
E_{0}(t)=\frac{1}{2 \pi} \sum_{k=0}^{N-1} c_{k+1}(-1)^{k+1} E^{(k)}(t), \quad \text { for any } t>0
$$

Therefore, since $c_{N} \neq 0$, it follows from Lemma 8.5 (ii) that the limit $E^{(N-1)}(0+)$ exists and $E_{0}(0+)=\frac{1}{2 \pi} c_{N}(-1)^{N} E^{(N-1)}(+0)$. On the other hand, by (8.2), Theorem 8.1 and Lemma 8.6 (i),

$$
E(t-s)=E(t) E_{0}(-s)+\sum_{n=1}^{N-1}(-1)^{n} E^{(n)}(t) E_{n}(-s), \quad \text { for any } s, t, s<0<t .
$$

Letting $s$ tend to zero, we see from Lemma 8.3 (iv) and $E(t)=E(t) E_{0}(0+)$ for any $t>0$. Thus, by (2.14), (2.15) and (2.16), $E_{0}(0+)=1$.

REMARK 8.3. Though by Lemmas 8.4, 8.5 (ii) and Proposition 8.1 the limits $E^{(n)}(0+)(n=N, N+1, \cdots)$ exist, they need not be always zero.

8.2. In this subsection we shall consider the case where $P$ is a transcendental function with an infinite number of roots. In this case we shall show

LEMMA 8.7. (i) $\left\{E^{(N)} ; n \in N_{*}\right\}$ is linearly independent in $\mathscr{A}((0, \infty))$,

(ii) $\left\{E^{(n)}, E_{n} ; n \in N_{*}\right\}$ is linearly independent in $\mathscr{A}((0, \infty))$.

Proof. For the proof of (i), it suffices to show that, for any $N \in N_{*}$ and any $a_{n} \in R(n=0,1, \cdots, N)$,

$$
\text { if } \sum_{n=0}^{N} a_{n} E^{(n)}(t)=0 \quad \text { for any } t>0, \text { then } a_{n}=0(n=0,1, \cdots, N) .
$$

We prove this by the method of mathematical induction on $N$. When $N$ is zero, it is clear by (2.14) and (2.15). Next we suppose for any fixed $N_{0} \geq 0$ that (8.13) holds for $N=N_{0}$ and consider any $a_{n} \in \boldsymbol{R}\left(n=0,1, \cdots, N_{0}+1\right)$ such that

$$
\sum_{n=0}^{N_{0}+1} a_{n} E^{(n)}(t)=0 \quad \text { in }(0, \infty) .
$$

Then, since by Lemma 8.3 (iii) 


$$
\left(-2 \pi c_{0}^{-1} a_{0} E_{0}(t)+\sum_{n=1}^{N_{0}+1} a_{n} E^{(n-1)}(t)\right)^{\prime}=0 \quad \text { in }(0, \infty),
$$

there exists some constant $b_{0}$ such that

$$
-2 \pi c_{0}^{-1} a_{0} E_{0}(t)+\sum_{n=1}^{N_{0}+1} a_{n} E^{(n-1)}(t)=b_{0} \quad \text { in }(0, \infty)
$$

Furthermore, since by Lemma 8.3 (iii) this implies that for any $t>0$

$$
\left\{-2 \pi c_{0}^{-1}\left(c_{0}^{-1} c_{1} a_{0}+a_{1}\right) E_{0}(t)+2 \pi c_{0}^{-1} a_{0} E_{1}(t)+\sum_{n=2}^{I V_{0}+1} a_{n} \mathbb{E}^{(n-2)}(t)\right\}^{\prime}=b_{0}
$$

there exists some constant $b_{1}$ such that for any $t>0$

$$
-2 \pi c_{0}^{-1}\left(c_{0}^{-1} c_{1} a_{0}+a_{1}\right) E_{0}(t)+2 \pi c_{0}^{-1} a_{0} E_{1}(t)+\sum_{n=2}^{N N_{0}+1} a_{n} E^{(n-2)}(t)=b_{0}+b_{1} t .
$$

Repeating this procedure, we can prove that there exist $d_{n} \in \mathbb{R}(n=$ $0,1, \cdots, N_{0}$ ) and a polynomial $q_{1}$ of order $N_{0}$ such that

$$
\sum_{n=0}^{N_{0}} d_{n} E_{n}(t)+a_{N_{0}+1} E(t)=q_{1}(t) \quad \text { in }(0, \infty) .
$$

By (2.17) and Lemma 8.3 (i), this implies that $q_{1}(\cdot)=0$. Therefore, it follows from (8.1) and Lemma 8.2 that

$$
\left(\sum_{n=0}^{N_{0}} d_{n} \frac{P(-\lambda)-\sum_{k=0}^{n} c_{k}(i \lambda)^{k}}{(i \lambda)^{n+1}}+2 \pi a_{N_{0}+1}\right) h(\lambda)=0 \quad \text { in } \boldsymbol{R} .
$$

Furthermore, by (2.14), this implies that there exists a polynomial $q_{2}$ of at most order $N_{0}$ such that

$$
\left(\sum_{n=0}^{N_{0}} d_{n}(i \lambda)^{N_{0}-n}\right) P(-\lambda)=2 \pi a_{N_{0}+1}+q_{2}(\lambda) \quad \text { in } C .
$$

Since $P$ has an infinite number of roots and $q_{2}$ has at most $N_{0}$ roots, we see that $\sum_{n=0}^{N_{0}} d_{n}(i \lambda)^{N_{0}-n}=0$ and hence $\alpha_{N_{0}+1}=0$. Thus, by (8.14) and the assumption of the method of mathematical induction, we have $a_{n}=0\left(n=0,1, \cdots, N_{0}+1\right)$. This completes the proof of (8.13). Next, we shall show (ii). For this, it suffices to prove that for any $N, M \in N_{*}$ and any $a_{n}, b_{m} \in \boldsymbol{R}(n=0,1, \cdots, N$, $m=0,1, \cdots, M)$ if

$$
\sum_{n=0}^{N} a_{n} E^{(n)}(t)+\sum_{m=0}^{M} b_{m} E_{m}(t)=0 \quad \text { in }(0, \infty),
$$


then $a_{n}=b_{m}=0(n=0,1, \cdots, N, m=0,1, \cdots, M)$. When $M$ is zero, (8.15) becomes

$$
\sum_{n=0}^{N} a_{n} E^{(n)}(t)+b_{0} E_{0}(t)=0 \quad \text { in }(0, \infty)
$$

By differentiating this and noting Lemma 8.3 (iii)

$$
\sum_{n=0}^{N} a_{n} E^{(n+1)}(t)-\frac{c_{0} b_{0}}{2 \pi} E(t)=0 \quad \text { in }(0, \infty) .
$$

Since $c_{0} \neq 0$, we see from (8.13) that $a_{n}=b_{0}=0(n=0,1, \cdots N)$. Therefore, we suppose that $M \geq 1$. Then, by Lemma 8.3 (iii), we note that there exist real constants $b_{l}^{m}(m=0,1, \cdots, M, l=1, \cdots, M)$ for which

$$
\begin{aligned}
& E_{m}^{(M+1)}=\sum_{l=1}^{M} b_{l}^{m} E^{(l)} \quad \text { in }(0, \infty) \quad(m=0,1, \cdots, M-1), \\
& E_{M}^{(M+1)}=\frac{c_{0}}{2 \pi} E+\sum_{l=1}^{M} b_{l}^{M} E^{(l)} \quad \text { in }(0, \infty) .
\end{aligned}
$$

By differentiating (8.15) $M+1$-times, we see from (8.16) that

$$
\sum_{n=0}^{N} a_{n} E^{(n+M+1)}(t)+\frac{c_{0} b_{M}}{2 \pi} E(t)+\sum_{l=1}^{M}\left(\sum_{m=0}^{M} b_{m} b_{l}^{m}\right) E^{(l)}(t)=0 \quad \text { in }(0, \infty) .
$$

Since $c_{0} \neq 0$, it follows from (8.13) that $b_{M}=0$. Therefore, we have $b_{m}=0$ $(m=0,1, \cdots, M)$ by the method of mathematical induction on $M$. Using (8.13) again, we have $a_{n}=0(n=0,1, \cdots, N)$. Thus, we have proved Lemma 8.7.

(Q.E.D.)

In concluding this section, we shall arrange the results which we have proved. We define two sequences $\left(f_{n}\right)_{n=0}^{\infty}$ and $\left(g_{n}\right)_{n=0}^{\infty}$ of elements in $\mathscr{A}(\boldsymbol{R}-\{0\})$ by

$$
f=(-1)^{n} E^{(n)} \text { and } g_{n}=\check{E}_{n}
$$

Then, combining (8.2), Theorem 8.1, Lemmas 8.4, 8.6 and 8.7, we have

THEOREM 8.2. (i ) For any $s \in(-\infty, 0)$ and any $t \in(0, \infty)$

$$
E(t-s)=\sum_{n=0}^{\infty} f_{n}(t) g_{n}(s)
$$

(ii) For any $n \in N_{*}$

$P\left(\frac{1}{i} \frac{d}{d t}\right) f_{n}(t)=0 \quad$ in $(0, \infty)$ and $\check{P}\left(\frac{1}{i} \frac{d}{d s}\right) g_{n}(s)=0 \quad$ in $(-\infty, 0)$.

(iii) If $P$ is a polynomial of order $N$, then 
(a) $g_{n}=0$, for any $n \in\{N, N+1, \cdots\}$,

(b) $\left\{f_{n} ; n=0,1, \cdots, N-1\right\}$ is linearly independent in $L^{2}(\boldsymbol{R})$,

(c) $\left\{g_{n} ; n=0,1, \cdots, N-1\right\}$ is linearly independent in $L^{2}(\boldsymbol{R})$.

(iv) If $P$ is a transcendental function with an infinite number of roots, then, $\left\{f_{n}, g_{n} ; n \in N_{*}\right\}$ is linearly independent in $\mathscr{A}(\boldsymbol{R}-\{0\})$.

REMARK 8.4. By Theorem 8.2, we might say that the canonical representation kernel $\sqrt{2 \pi}-1$ is a "modified" Goursat kernel also in a general case where $P$ need not be always a polynomial ([7]).

REMARK 8.5. We assume that $P$ is a polynomial of order $N$. It then follows from Theorem 8.2 (ii) (iii) that $\left\{f_{n}, g_{n} ; n=0,1, \cdots, N-1\right\}$ is linearly dependent in $\mathscr{A}(\boldsymbol{R}-\{0\})$.।

\section{§ 9. Germ space (II)}

Similary as in $\S 8$, we shall assume that $\boldsymbol{X}$ has ths Markovian property and $E$ is real analytic in $(0, \infty)$. In $\S 8$ we have obtained a formula for $E$ and analysed its structure on the space $L^{2}(R)$. In this section, applying the results in $\S 8$ to the space $M$, which is isometric to $L^{2}(R)$ (Propositions 2.1 and 2.5), we shall investigate the structure of the spaces $M(D)$ ( $D$ open set in $\boldsymbol{R})$ and $\partial \boldsymbol{M}(t)(t \in \boldsymbol{R})$ as a continuation of $\S 6$.

By means of Lemma 8.3 (i), we can define a sequence of real meancontinuous stationary Gaussion processes $\boldsymbol{Y}_{n}=\left(y_{n}(t) ; t \in \boldsymbol{R}\right)$ with representation kernels $\sqrt{2 \pi}^{-1} E_{n}\left(n \in N_{*}\right)$ :

$$
y_{n}(t)=\int_{R} \sqrt{2 \pi}^{-1} E_{n}(t-s) d B(s)
$$

Furthermore, using these processes, we introduce a stochastic process $\mathscr{Y}=$ $(\mathscr{Y}(t) ; t \in \boldsymbol{R})$ whose state spaces is an infinite-dimensional Euclidean space $\boldsymbol{R}^{\infty}$ and $n$-th component process is $\boldsymbol{Y}_{n-1}(n \in N)$ :

$$
\mathscr{Y}(t)=\left(y_{0}(t), y_{1}(t), \cdots\right) \text {. }
$$

In the same way as for $\boldsymbol{X}$, we define several sub- $\sigma$-fields of $\boldsymbol{F}$ which are associated with $\mathscr{Y}$.

Definition 9.1. For any open set $D$ in $\boldsymbol{R}$ and any $a \in \boldsymbol{R}$, we set

$$
\begin{aligned}
& \boldsymbol{F}_{\mathbf{g}}(D)=\sigma(\{\mathscr{Y}(t) ; t \in D\}), \\
& \partial \boldsymbol{F}_{\mathrm{g}}(a)=\bigcap_{\mathrm{s}>0} \boldsymbol{F}_{\mathrm{g}}((a-\varepsilon, a+\varepsilon)), \quad \boldsymbol{F}_{\mathrm{y}}^{+}(a)=\boldsymbol{F}_{\mathrm{y}}((a, \infty)), \quad \boldsymbol{F}_{\mathrm{g}}^{-}(a)=\boldsymbol{F}_{\mathrm{y}}((-\infty, a)) \text { and } \\
& \boldsymbol{F}_{\mathbb{9}}^{+/-}(a)=\text { the smallest splitting field of } \boldsymbol{F}_{\mathbb{g}}^{+}(a) \text { upon } \boldsymbol{F}_{\mathbb{g}}^{-}(a) \text {. }
\end{aligned}
$$

At first, in connection with the continuity of path functions of $\mathscr{Y}$, we shall show 
THEOREM 9.1. (i) For almost all $\omega \in \Omega$,

(a) each $y_{n}(t)\left(n \in \mathbb{N}_{*}\right)$ is continuous in $t \in \boldsymbol{R}$,

(b) $y_{0}(t)$ is not differentiable at almost everywhere $t \in \boldsymbol{R}$,

(c) each $y_{n}(t)(n \in N)$ is differentiable at almost everywhere $t \in \boldsymbol{R}$.

(ii) (a) $y_{0}(t)$ is not differentiable in the mean,

(b) for any $n \in N, y_{n}(t)$ is differential in the mean and $y_{n}^{(1)}=-y_{n-1}(t)$ $-\frac{c_{n}}{2 \pi} X(t)$.

Proof. Since by Lemma 8.3 (i) (iv) $\chi_{(0, \infty)} E_{n}$ is differentiable in $(0, \infty)$ and its derivative belongs to $L^{2}((0, \infty))\left(n \in N_{*}\right)$, for any $n \in N_{*}$ and any $s, t \in \boldsymbol{R}, s<t$,

$$
\begin{aligned}
\sqrt{2 \pi}\left(y_{n}(t)-y_{n}(s)\right) & =\int_{s}^{t} E_{n}(t-u) d B(u)+\int_{-\infty}^{s}\left(E_{n}(t-u)-E_{n}(s-u)\right) d B(u) \\
& =\int_{s}^{t} E_{n}(t-u) d B(u)+\int_{-\infty}^{s}\left(\int_{s}^{t} E_{n}^{(1)}(v-u) d v\right) d B(u) \\
& =\int_{s}^{t} E_{n}(t-u) d B(u)+\int_{s}^{t}\left(\int_{-\infty}^{s} E_{n}^{(1)}(v-u) d B(u)\right) d v
\end{aligned}
$$

Therefore, for $n=0$, it follows from Lemmas 6.1 and 8.3 (i) (iii) (iv) that, for any $s, t \in \boldsymbol{R}, s<t$,

$$
\begin{aligned}
\sqrt{2 \pi}\left(y_{0}(t)-y_{0}(s)\right)= & E_{0}(0+)(B(t)-B(s))+\int_{s}^{t}\left(E_{0}(t-u)-E_{0}(0+)\right) d B(u) \\
& +\int_{s}^{t}\left(\int_{-\infty}^{s} E_{0}^{(1)}(v-u) d B(u)\right) d v \\
= & E_{0}(0+)(B(t)-B(s))+\int_{s}^{t}\left(\int_{s}^{v} E_{0}^{(\mathrm{i})}(v-u) d B(u)\right) d v \\
& +\int_{s}^{t}\left(\int_{-\infty}^{s} E_{0}^{(1)}(v-u) d B(u)\right) d v \\
= & E_{0}(0+)(B(t)-B(s))-\frac{c_{0}}{2 \pi} \int_{s}^{t}\left(\int_{-\infty}^{v} E(v-u) d B(u)\right) d v \\
= & E_{0}(0+)(B(t)-B(s))-\frac{c_{0}}{2 \pi} \int_{s}^{t} X(v) d v .
\end{aligned}
$$

Similarly, for any $n \in N$ and any $s, t \in R, s<t$,

$$
\sqrt{2 \pi}\left(y_{n}(t)-y_{n}(s)\right)=\int_{s}^{t}\left(\int_{-\infty}^{v} E_{n}^{(1)}(v-u) d B(u)\right) d v
$$

Now, since for almost all $\omega \in \Omega$ and any $n \in N X(v)$ and $\int_{-\infty}^{v} E_{n}^{(1)}(v-u) d B(u)$ are locally integrable in $v \in \boldsymbol{R}$, (i) (a) and (i) (c) follow from (9.3) and (9.4). 
Since for almost all $\omega \in \Omega B(t)$ is not differentiable in $t \in \mathbb{R}$, we have (i) (b) by (9.3) and the fact $E_{0}(0+) \neq 0$. Moreover, since $\|B(t)-B(s)\|_{M}=\sqrt{t-s}$ and $\left\|\int_{s}^{t} X(v) d v\right\|_{M} \leqslant \sqrt{k(0)}(t-s)$, we have (ii) (a) by (9.3) and the fact $E_{0}(0+)$ $\neq 0$. Finally we shall show (ii) (b). Fix any $n \in N$. It follows from (8.2), Lemma 8.3 (i) (iii) and (9.4) that, for any $s, t \in \boldsymbol{R}, s<t$,

$$
\begin{aligned}
& \sqrt{2 \pi} \frac{y_{n}(t)-y_{n}(s)}{t-s}-\int_{-\infty}^{s} E_{n}^{(1)}(s-u) d B(u) \\
& =\frac{1}{s-t} \int_{s}^{t}\left[\int_{R}\left(\varphi_{n}(v-u)-\varphi_{n}(s-u)\right) d B(u)\right] d v,
\end{aligned}
$$

where $\varphi_{n}$ is an $L^{2}$-function defined by $\varphi_{n}=E_{n-1}+\frac{c_{n}}{2 \pi} E$. Therefore, since

$$
\left\|\sqrt{2 \pi} \frac{y_{n}(t)-y_{n}(s)}{t-s}-\int_{-\infty}^{s} E_{n}^{(1)}(s-u) d B(u)\right\|_{M}^{2} \leqslant \frac{1}{t-s} \int_{s}^{t}\left\|\varphi_{n}(v-s+\cdot)-\varphi_{n}(\cdot)\right\|_{L^{2}}^{2} d v
$$

we see from Lemmas 6.1, 8.3 (i) (iii) and (9.1) that $\lim _{t \rightarrow s} \cdot \frac{y_{n}(t)-y_{n}(s)}{t-s}$ exists and it equals $-y_{n-1}(s)-\frac{c_{n}}{2 \pi} X(s)$.

Next, in connection with the structure of the spaces $\partial M(t)(t \in \boldsymbol{R})$ and $\boldsymbol{M}(D)$ ( $D$ open set in $\boldsymbol{R})$, we shall show

THEOREM 9.2. (i ) $\left\{y_{n}(t) ; n \in N_{*}\right\} \subset \partial M(t)$, for any $t \in \boldsymbol{R}$.

(ii) $\boldsymbol{F}(D)=\boldsymbol{F}_{y}(D)$, for any open set $D$ in $\boldsymbol{R}$.

(iii) $\boldsymbol{F}^{+/-}(t)=\partial \boldsymbol{F}(t)=\boldsymbol{F}_{8}^{+/-}(t)=\partial \boldsymbol{F}_{8}(t)$, for any $t \in \boldsymbol{R}$.

(iv) If $\boldsymbol{P}$ is a polynomial of order $N$, then

(a) $\boldsymbol{Y}_{n}=0$, for any $n \in\{N, N+1, \cdots\}$,

(b) $\left\{\boldsymbol{Y}_{0}, \boldsymbol{Y}_{1}, \cdots, \boldsymbol{Y}_{N-1}\right\}$ is linearly independent in the sense that for any fixed $t \in \boldsymbol{R}$, if $\sum_{n=0}^{N-1} a_{n} y_{n}(t)=0$, then $a_{n}=0(n=0,1, \cdots, N-1)$,

(c) $\partial M(t)=$ the closed linear hull of $\left\{y_{n}(t) ; n=0,1, \cdots, N-1\right\}$, for any $t \in \boldsymbol{R}$.

(v) If $P$ is a transcendental function with an infinite number of roots, then $\left\{\boldsymbol{Y}_{n} ; n \in \boldsymbol{N}_{*}\right\}$ is linearly independent in the sense that, for any $N \in N$ and any fixed $t \in \boldsymbol{R}$, if $\sum_{n=0}^{N} a_{n} y_{n}(t)=0$, then $a_{n}=0(n=0,1, \cdots, N)$.

Proof. Fix any $n \in N_{*}$ and any $t \in R$. By Lemma 8.2,

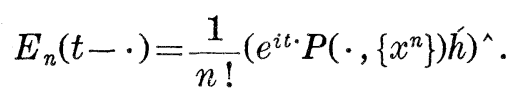

Therefore, since $e^{i t \cdot} P\left(\cdot,\left\{x^{n}\right\}\right) \in \partial Z_{4}(t)$ by Definition 2.6 and Lemma 8.1 (i), 
it follows from Corollary 2.1, Proposition 2.5, Lemma 2.15 and (9.1) that $y_{n}(t) \in \partial \boldsymbol{M}(t)$. This implies (i). Fix any open set $D$ in $\boldsymbol{R}$. Since each process $\boldsymbol{Y}_{n}$ is Gaussian, it follows from (i) and Lemma 2.1 (i) that $\boldsymbol{F}_{8}(D) \subset$ $F(D)$. On the other hand, since by (2.13), (7.3) and (7.6) there exists some $n \in \boldsymbol{N}$ such that $c_{n} \neq 0$, it follows from Theorem 9.1 (ii) (b) that $\boldsymbol{F}(D) \subset \boldsymbol{F}_{y}(D)$. Therefore, we have (ii). Since $\partial \boldsymbol{F}(t)=\boldsymbol{F}^{+/-}(t)$ by Lemma 2.1 (i) (iii), Corollary 2.1, Lemma 2.4 and Proposition 2.2, it is clear that (ii) implies (iii). Next we shall show (iv). By (8.17) and (9.1), (iv) (a) and (iv) (b) follow from (iii) (a) and (iii) (c) in Theorem 8.2, respectively. (iv) (c) can be proved as follows: Fix any $u>t$. By Lemma 6.1, (8.17), Theorem 8.2 (i), (iii) (a) and (9.1),

$$
\begin{aligned}
& E\left(X(u) \mid \boldsymbol{F}^{-}(t)\right)=\int_{-\infty}^{t} \sqrt{2 \pi^{-1}} E(u-s) d B(s) \\
& =\sum_{n=0}^{N-1} f_{n}(u-t) \int_{-\infty}^{t} \sqrt{2 \pi^{-1}} g_{n}(s-t) d B(s) \\
& =\sum_{n=0}^{N-1} f_{n}(u-t) y_{n}(t) \text {. }
\end{aligned}
$$

Therefore, it follows from Lemmas 2.1, 2.4 and Proposition 2.2 that $\partial \boldsymbol{M}(t) \subset$ the closed linear hull of $\left\{y_{n}(t) ; n=0,1, \cdots, N-1\right\}$. Combining this with (i), we have (iv) (c). Finally, (v) follows from (8.17), Theorem 8.2 (iv) and (9.1)

(Q.E.D.)

REMARK 9.1. We assume that $P$ is a polynomial of order $N$. Similarly as in Theorem 9.1 (ii), it follows from (6.1) and Lemma 8.5 (ii) that $X(t)$ is differentiable up to $N-1$ times in the mean and its derivatives satisfy

$$
X^{(n)}(t)=\int_{R} \sqrt{2 \pi}^{-1} E^{(n)}(t-s) d B(s) \quad(n=0,1, \cdots, N-1) .
$$

Therefore, by (8.2), (8.17), (9.1), (9.5) and (9.6), for any $t>0$,

$$
E\left(X(t) \mid F^{-}(0)\right)=\frac{1}{2 \pi} \sum_{n=0}^{N-1}\left(\sum_{k=0}^{N-n-1} c_{n+k+1}(-1)^{n+k+1} E^{(k)}(t)\right) X^{(n)}(0) .
$$

Thus, combining Corollary 7.2 with (9.7), we have proved (6.15).

\section{References}

[1] L. Arnold: Eine Bemerkung zur "Quadramittel-Funktionentheorie," Arch. Math. 17 (1966), 544-547.

[2] N. Aronszajn: Theory of reproducing kernels, Trans. Amer. Math. Soc. 68 (1950), 337-404. 
[ 3 ] L. de Branges: Local operators on Fourier transforms, Duke Math. J. 25 (1958), 143-154.

[ 4 ] L. de Branges: Hilbert spaces of entire functions, Prentice Hall, Inc., Englewood Cliffs, N.J., 1968.

[5] J.L. Doob: The elementary Gaussian processes, Ann. Math. Statist. 15 (1944), 229-282.

[6] H. Dym and H.P. McKean, Jr.: Application of de Branges spaces of integral functions to the prediction of stationary Gaussian processes, Illinois J. Math. 45 (1970), 299-343.

[ 7 ] T. Hida: Canonical representations of Gaussian processes and their applications, Mem. Coll. Sci. Univ. Kyoto, Ser. A, Math. 34 (1960), 109-155.

[8] E. Hille and J.D. Tamarkin: On a theorem of Paley and Wiener, Ann. of Math. 34 (1933), 606-614.

[9] K. Hoffman: Banach spaces of analytic functions, Prentice Hall, Englewood Cliffs, N.J., 1962.

[10] K. Itô: Stationary random distributions, Mem. Coll. Sci. Univ. Kyoto, Ser. A, Math. 28 (1954), 209-223.

[11] K. Karhunen: Über die struktur stationärer zufälliger funktionnen, Ark. Mat. $1(1950), 141-160$.

[12] T. Kawai: On the theory of Fourier hyperfunctions and its applications to partial differential equations with constant coefficients, J. Fac. Sci. Univ. Tokyo 17 (1971), 467-517.

[13] H. Komatsu: Projective and injective limits of weakly compact sequences of locally convex spaces, J. Math. Soc. Japan 19 (1967), 366-383.

[14] N. Levinson and H.P. McKean, Jr.: Weighted trigonometrical approximation on $\boldsymbol{R}^{1}$ with application to the germ field of a stationary Gaussian noise, Acta Math. 112 (1964), 99-143.

[15] H.P. McKean, Jr.: Brownian motion with a several-dimensional time, Teor. Veroyatnost. i Primenen. 8 (1963), 357-378.

[16] Y. Okabe: Markovian property of stationary Gaussian processes and M. Sato's hyperfunctions, Sûrikaisekikenkyûsho Kokyûroku 145, R.I.M.S., Kyoto Univ., 1972, 48-60 (in Japanese).

[17] R. E. A. C. Paley and N. Wiener: Fourier transforms in the complex domain, AMS Colloquim Publications 19, Providence R.I.

[18] M. Sato: Chôkansû no Riron, Sûgaku 10 (1958), 1-27 (in Japanese).

[19] M. Sato: Theory of hyperfunctions, I, J. Fac. Sci. Univ. Tokyo Sec. I 8 (1959), 139-193.

[20] M. Sato: Theory of hyperfunctions, II, J. Fac. Sci. Univ. Tokyo Sec. I 8 (1959), 387-437.

[21] M. Sato: Hyperfunctions and partial differential equations, Proc. Intern. Conf. on Functional Analysis and Related Topics, Tokyo, 1969, Univ. of Tokyo Press, 1970, 91-94.

[22] M. Sato: On the structure of hyperfunctions, Sûgaku no Ayumi 15 (1970), 9-72 (notes by M. Kashiwara, in Japanese).

[23] L. Schwartz: Theorie des distributions, I, II, Hermann, Paris, 1951-1952.

[24] L. Schwartz: Sous-espaces hilbertiens d'espaces vectoriels topologiques et noyaux associes, J. Analyse Math. 13 (1964), 115-256.

[25] V.N. Tutubalin and M.I. Freidlin: On the structure of the infinitesimal $\sigma$-algebra of a Gaussian process, Teor. Veroyatnost. i Primenen. 7 (1962), 196-199. 
[26] K. Urbanik: Generalized stationary processes of Markovian character, Studia Math. 21 (1962), 261 282.

[27] N. Wiener: Extrapolation, interpolation and smoothing of stationary time series, Wiley, New York, 1949.

Department of Mathematics FACULTY OF SCIENCE OSAKA UNIVERSITY

TOYONAKA, OSAKA, JAPAN 